\title{
Inclusive measurement of diffractive deep-inelastic scattering at HERA
}

The H1 Collaboration

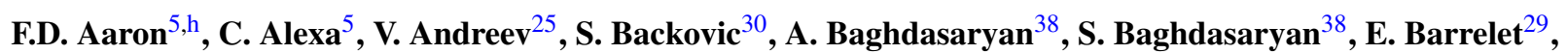
W. Bartel ${ }^{11}$, K. Begzsuren ${ }^{35}$, A. Belousov ${ }^{25}$, P. Belov ${ }^{11}$, J.C. Bizot ${ }^{27}$, V. Boudry ${ }^{28}$, I. Bozovic-Jelisavcic ${ }^{2}$, J. Bracinik ${ }^{3}$, G. Brandt ${ }^{11}$, M. Brinkmann ${ }^{11}$, V. Brisson ${ }^{27}$, D. Britzger ${ }^{11}$, D. Bruncko ${ }^{16}$, A. Bunyatyan ${ }^{13,38}$, A. Bylinkin ${ }^{24}$, L. Bystritskaya ${ }^{24}$, A.J. Campbell ${ }^{11}$, K.B. Cantun Avila $^{22}$, F. Ceccopieri ${ }^{4}$, K. Cerny ${ }^{32}$, V. Cerny ${ }^{16, \mathrm{~g}}$, V. Chekelian ${ }^{26}$, J.G. Contreras ${ }^{22}$, J.A. Coughlan ${ }^{6}$, J. Cvach $^{31}$, J.B. Dainton ${ }^{18}$, K. Daum ${ }^{37, a, c}$, B. Delcourt ${ }^{27}$, J. Delvax ${ }^{4}$, E.A. De Wolf ${ }^{4}$, C. Diaconu ${ }^{21}$, M. Dobre ${ }^{12, j, k}$, V. Dodonov ${ }^{13}$, A. Dossanov ${ }^{26}$, A. Dubak ${ }^{30, f}$, G. Eckerlin ${ }^{11}$, S. Egli ${ }^{36}$, A. Eliseev ${ }^{25}$, E. Elsen ${ }^{11}$, L. Favart ${ }^{4}$, A. Fedotov ${ }^{24}$, R. Felst ${ }^{11}$, J. Feltesse ${ }^{10}$, J. Ferencei ${ }^{16}$, D.-J. Fischer ${ }^{11}$, M. Fleischer ${ }^{11}$, A. Fomenko ${ }^{25}$, E. Gabathuler ${ }^{18}$, J. Gayler ${ }^{11}$, S. Ghazaryan ${ }^{11}$, A. Glazov ${ }^{11}$, L. Goerlich ${ }^{7}$, N. Gogitidze ${ }^{25}$, M. Gouzevitch ${ }^{11, e}$, C. Grab ${ }^{40}$, A. Grebenyuk ${ }^{11}$, T. Greenshaw ${ }^{18}$, G. Grindhammer ${ }^{26}$, S. Habib $^{11}$, D. Haidt ${ }^{11}$, R.C.W. Henderson ${ }^{17}$, E. Hennekemper ${ }^{15}$, H. Henschel ${ }^{39}$, M. Herbst ${ }^{15}$, G. Herrera ${ }^{23}$, M. Hildebrandt ${ }^{36}$, K.H. Hiller ${ }^{39}$, J. Hladký ${ }^{31}$, D. Hoffmann ${ }^{21}$, R. Horisberger ${ }^{36}$, T. Hreus ${ }^{4, d}$, F. Huber ${ }^{14}$, M. Jacquet ${ }^{27}$, X. Janssen ${ }^{4}$, L. Jönsson ${ }^{20}$, H. Jung ${ }^{11,4}$, M. Kapichine ${ }^{9}$, I.R. Kenyon ${ }^{3}$, C. Kiesling ${ }^{26}$, M. Klein ${ }^{18}$, C. Kleinwort ${ }^{11}$, T. Kluge ${ }^{18}$, R. Kogler ${ }^{12}$, P. Kostka ${ }^{39}$, M. Krämer ${ }^{11}$, J. Kretzschmar ${ }^{18}$, K. Krüger ${ }^{15}$, M.P.J. Landon ${ }^{19}$, W. Lange ${ }^{39}$,

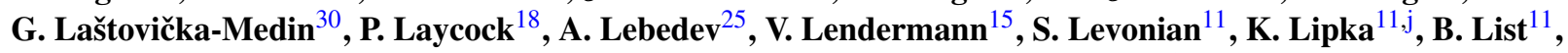
J. List ${ }^{11}$, B. Lobodzinski ${ }^{11}$, R. Lopez-Fernandez ${ }^{23}$, V. Lubimov ${ }^{24}$, E. Malinovski ${ }^{25}$, H.-U. Martyn ${ }^{1}$, S.J. Maxfield ${ }^{18}$, A. Mehta ${ }^{18}$, A.B. Meyer $^{11}$, H. Meyer ${ }^{37}$, J. Meyer ${ }^{11}$, S. Mikocki ${ }^{7}$, I. Milcewicz-Mika ${ }^{7}$, F. Moreau ${ }^{28}$, A. Morozov ${ }^{9}$, J.V. Morris ${ }^{6}$, K. Müller ${ }^{41}$, Th. Naumann ${ }^{39}$, P.R. Newman ${ }^{3}$, C. Niebuhr ${ }^{11}$, D. Nikitin ${ }^{9}$, G. Nowak ${ }^{7}$, K. Nowak ${ }^{11}$, J.E. Olsson ${ }^{11}$, D. Ozerov ${ }^{24}$, P. Pahl ${ }^{11}$, V. Palichik ${ }^{9}$, I. Panagoulias ${ }^{11, b, x}$, M. Pandurovic ${ }^{2}$, Th. Papadopoulou ${ }^{11, b, x}$,

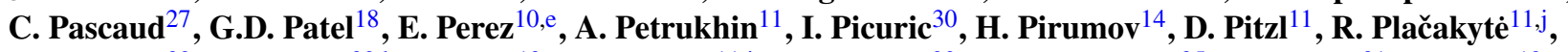
B. Pokorny ${ }^{32}$, R. Polifka ${ }^{32,1}$, B. Povh ${ }^{13}$, V. Radescu ${ }^{11, j}$, N. Raicevic ${ }^{30}$, T. Ravdandorj ${ }^{35}$, P. $\operatorname{Reimer}^{31}$, E. Rizvi ${ }^{19}$, P. Robmann ${ }^{41}$, R. Roosen $^{4}$, A. Rostovtsev ${ }^{24}$, M. Rotaru ${ }^{5}$, J.E. Ruiz Tabasco ${ }^{22}$, S. Rusakov $^{25}$, D. Š́lek ${ }^{32}$, D.P.C. Sankey ${ }^{6}$, M. Sauter ${ }^{14}$, E. Sauvan ${ }^{21, m}$, S. Schmitt ${ }^{11}$, L. Schoeffel ${ }^{10}$, A. Schöning ${ }^{14}$, H.-C. Schultz-Coulon ${ }^{15}$, F. Sefkow ${ }^{11}$, L.N. Shtarkov ${ }^{25}$, S. Shushkevich ${ }^{11}$, T. Sloan ${ }^{17}$, Y. Soloviev ${ }^{25}$, P. Sopicki ${ }^{7}$, D. South ${ }^{11}$, V. Spaskov ${ }^{9}$, A. Specka ${ }^{28}$, Z. Staykova ${ }^{4}$, M. Steder ${ }^{11}$, B. Stella ${ }^{33}$, G. Stoicea ${ }^{5}$ U. Straumann ${ }^{41}$, T. Sykora ${ }^{4,32}$, P.D. Thompson ${ }^{3}$, T.H. $\operatorname{Tran}^{27}$, D. Traynor $^{19}$, P. Truöl ${ }^{41}$, I. Tsakov ${ }^{34}$, B. Tseepeldorj ${ }^{35, i}$, J. Turnau ${ }^{7}$, A. Valkárová ${ }^{32}$, C. Vallée ${ }^{21}$, P. Van Mechelen ${ }^{4}$, Y. Vazdik ${ }^{25}$, D. Wegener ${ }^{8}$, E. Wünsch ${ }^{11}$, J. Žáček ${ }^{32}$, J. Zálešák ${ }^{31}$, Z. Zhang ${ }^{27}$, A. Zhokinn ${ }^{24}$, R. Žlebčík ${ }^{32}$, H. Zohrabyan ${ }^{38}$, F. Zomer ${ }^{27}$

\footnotetext{
${ }^{1}$ I. Physikalisches Institut der RWTH, Aachen, Germany

${ }^{2}$ Vinca Institute of Nuclear Sciences, University of Belgrade, 1100 Belgrade, Serbia

${ }^{3}$ School of Physics and Astronomy, University of Birmingham, Birmingham, $\mathrm{UK}^{\mathrm{o}}$

${ }^{4}$ Inter-University Institute for High Energies ULB-VUB, Brussels and Universiteit Antwerpen, Antwerpen, Belgium ${ }^{\mathrm{p}}$

${ }^{5}$ National Institute for Physics and Nuclear Engineering (NIPNE), Bucharest, Romania ${ }^{\mathrm{Z}}$

${ }_{7}^{6}$ STFC, Rutherford Appleton Laboratory, Didcot, Oxfordshire, $\mathrm{UK}^{\mathrm{O}}$

${ }^{7}$ Institute for Nuclear Physics, Cracow, Poland ${ }^{\mathrm{q}}$

${ }^{8}$ Institut für Physik, TU Dortmund, Dortmund, Germany ${ }^{\mathrm{n}}$

${ }^{9}$ Joint Institute for Nuclear Research, Dubna, Russia

${ }^{10} \mathrm{CEA}, \mathrm{DSM} / \mathrm{Irfu}, \mathrm{CE}-\mathrm{Saclay}$, Gif-sur-Yvette, France

${ }^{11}$ DESY, Hamburg, Germany

${ }^{12}$ Institut für Experimentalphysik, Universität Hamburg, Hamburg, Germany ${ }^{\mathrm{n}}$

${ }^{13}$ Max-Planck-Institut für Kernphysik, Heidelberg, Germany

${ }^{14}$ Physikalisches Institut, Universität Heidelberg, Heidelberg, Germany ${ }^{\mathrm{n}}$

${ }^{15}$ Kirchhoff-Institut für Physik, Universität Heidelberg, Heidelberg, Germany ${ }^{\mathrm{n}}$

${ }^{16}$ Institute of Experimental Physics, Slovak Academy of Sciences, Košice, Slovak Republic ${ }^{\mathrm{s}}$

${ }^{17}$ Department of Physics, University of Lancaster, Lancaster, UK ${ }^{\mathrm{O}}$

${ }^{18}$ Department of Physics, University of Liverpool, Liverpool, UK ${ }^{\mathrm{O}}$

${ }^{19}$ School of Physics and Astronomy, Queen Mary, University of London, London, $\mathrm{UK}^{\mathrm{o}}$
} 
${ }^{20}$ Physics Department, University of Lund, Lund, Sweden ${ }^{\mathrm{t}}$

${ }^{21}$ CPPM, Aix-Marseille Univ, CNRS/IN2P3, 13288 Marseille, France

${ }^{22}$ Departamento de Fisica Aplicada, CINVESTAV, Mérida, Yucatán, México ${ }^{\mathrm{y}}$

${ }^{23}$ Departamento de Fisica, CINVESTAV IPN, México City, México ${ }^{\mathrm{y}}$

${ }^{24}$ Institute for Theoretical and Experimental Physics, Moscow, Russia ${ }^{\mathrm{W}}$

${ }^{25}$ Lebedev Physical Institute, Moscow, Russia ${ }^{r}$

${ }^{26}$ Max-Planck-Institut für Physik, München, Germany

${ }^{27}$ LAL, Université Paris-Sud, CNRS/IN2P3, Orsay, France

${ }^{28}$ LLR, Ecole Polytechnique, CNRS/IN2P3, Palaiseau, France

${ }^{29}$ LPNHE, Université Pierre et Marie Curie Paris 6, Université Denis Diderot Paris 7, CNRS/IN2P3, Paris, France

${ }^{30}$ Faculty of Science, University of Montenegro, Podgorica, Montenegro ${ }^{\text {aa }}$

${ }^{31}$ Institute of Physics, Academy of Sciences of the Czech Republic, Praha, Czech Republic ${ }^{\mathrm{u}}$

${ }^{32}$ Faculty of Mathematics and Physics, Charles University, Praha, Czech Republic ${ }^{\mathrm{u}}$

${ }^{33}$ Dipartimento di Fisica Università di Roma Tre and INFN Roma 3, Roma, Italy

${ }^{34}$ Institute for Nuclear Research and Nuclear Energy, Sofia, Bulgaria ${ }^{\mathrm{r}}$

${ }^{35}$ Institute of Physics and Technology of the Mongolian Academy of Sciences, Ulaanbaatar, Mongolia

${ }^{36}$ Paul Scherrer Institut, Villigen, Switzerland

${ }^{37}$ Fachbereich C, Universität Wuppertal, Wuppertal, Germany

${ }^{38}$ Yerevan Physics Institute, Yerevan, Armenia

${ }^{39}$ DESY, Zeuthen, Germany

${ }^{40}$ Institut für Teilchenphysik, ETH, Zürich, Switzerland ${ }^{\vee}$

${ }^{41}$ Physik-Institut der Universität Zürich, Zürich, Switzerland ${ }^{\mathrm{V}}$

Received: 20 March 2012 / Revised: 20 April 2012 / Published online: 18 July 2012

(C) The Author(s) 2012. This article is published with open access at Springerlink.com

\begin{abstract}
The diffractive process $e p \rightarrow e X Y$, where $Y$ denotes a proton or its low mass excitation with $M_{Y}<$ $1.6 \mathrm{GeV}$, is studied with the $\mathrm{H} 1$ experiment at HERA. The analysis is restricted to the phase space region of the photon virtuality $3 \leq Q^{2} \leq 1600 \mathrm{GeV}^{2}$, the square of the fourmomentum transfer at the proton vertex $|t|<1.0 \mathrm{GeV}^{2}$ and the longitudinal momentum fraction of the incident proton carried by the colourless exchange $x_{\mathbb{P}}<0.05$.
\end{abstract}

\footnotetext{
a e-mail: daum@mail.desy.de

${ }^{\mathrm{b}}$ Also at Physics Department, National Technical University, Zografou Campus, GR-15773 Athens, Greece

${ }^{\mathrm{c}}$ Also at Rechenzentrum, Universität Wuppertal, Wuppertal, Germany

${ }^{\mathrm{d}}$ Also at University of P.J. Šafárik, Košice, Slovak Republic

${ }^{\mathrm{e}}$ Also at CERN, Geneva, Switzerland

${ }^{\mathrm{f}}$ Also at Max-Planck-Institut für Physik, München, Germany

${ }^{\mathrm{g}}$ Also at Comenius University, Bratislava, Slovak Republic

${ }^{\mathrm{h}}$ Also at Faculty of Physics, University of Bucharest, Bucharest, Romania
}

${ }^{\mathrm{i}}$ Also at Ulaanbaatar University, Ulaanbaatar, Mongolia

${ }^{\mathrm{j} S u p p o r t e d ~ b y ~ t h e ~ I n i t i a t i v e ~ a n d ~ N e t w o r k i n g ~ F u n d ~ o f ~ t h e ~ H e l m h o l t z ~}$ Association (HGF) under the contract VH-NG-401 and SO-072

${ }^{\mathrm{k}}$ Absent on leave from NIPNE-HH, Bucharest, Romania

${ }^{\mathrm{l}}$ Also at Department of Physics, University of Toronto, Toronto, Ontario, Canada M5S 1A7

${ }^{\mathrm{m}}$ Also at LAPP, Université de Savoie, CNRS/IN2P3, Annecy-le-Vieux, France

${ }^{\mathrm{n}}$ Supported by the Bundesministerium für Bildung und Forschung, FRG, under contract numbers 05H09GUF, 05H09VHC, 05H09VHF, 05H16PEA
Triple differential cross sections are measured as a function of $x_{\mathbb{P}}, Q^{2}$ and $\beta=x / x_{\mathbb{P}}$ where $x$ is the Bjorken scaling variable. These measurements are made after selecting diffractive events by demanding a large empty rapidity interval separating the final state hadronic systems $X$ and $Y$. High statistics measurements covering the data taking periods 1999-2000 and 2004-2007 are combined with previously published results in order to provide a single set of diffractive cross sections from the $\mathrm{H} 1$ experiment using the

${ }^{\circ}$ Supported by the UK Science and Technology Facilities Council, and formerly by the UK Particle Physics and Astronomy Research Council pSupported by FNRS-FWO-Vlaanderen, IISN-IIKW and IWT and by Interuniversity Attraction Poles Programme, Belgian Science Policy

qPartially Supported by Polish Ministry of Science and Higher Education, grant DPN/N168/DESY/2009

${ }^{\mathrm{r}}$ Supported by the Deutsche Forschungsgemeinschaft

${ }^{\text {s}}$ Supported by VEGA SR grant no. 2/7062/ 27

${ }^{\text {t}}$ Supported by the Swedish Natural Science Research Council

"Supported by the Ministry of Education of the Czech Republic under the projects LC527, INGO-LA09042 and MSM0021620859

${ }^{\mathrm{v}}$ Supported by the Swiss National Science Foundation

${ }^{\mathrm{w}}$ Russian Foundation for Basic Research (RFBR), grant no 1329.2008.2 and Rosatom

${ }^{\mathrm{x}}$ This project is co-funded by the European Social Fund $(75 \%)$ and National Resources $(25 \%)$ - (EPEAEK II) - PYTHAGORAS II

${ }^{\mathrm{y}}$ Supported by CONACYT, México, grant 48778-F

${ }^{\mathrm{z}}$ Supported by the Romanian National Authority for Scientific Research under the contract PN 09370101

${ }^{\text {aa }}$ Partially Supported by Ministry of Science of Montenegro, no. 051/3-3352 
large rapidity gap selection method. The combined data represent a factor between three and thirty increase in statistics with respect to the previously published results. The measurements are compared with predictions from NLO QCD calculations based on diffractive parton densities and from a dipole model. The proton vertex factorisation hypothesis is tested.

\section{Introduction}

At HERA a substantial fraction of up to $10 \%$ of $e p$ interactions proceed via the diffractive scattering process initiated by a highly virtual photon [1-15]. In contrast to the standard deep inelastic scattering (DIS) process $e p \rightarrow e X$ (Fig. 1a), the diffractive reaction $e p \rightarrow e X Y$ contains two distinct final state systems (Fig. 1b), where $X$ is a high-mass hadronic state and $Y$ is the elastically scattered proton or its low-mass excitation, emerging from the interaction with almost the full energy of the incident proton.

The study and interpretation of diffraction at HERA provides essential inputs for the understanding of quantum chromodynamics (QCD) at high parton densities. The sensitivity of the diffractive cross section to the gluon density at low values of Bjorken $x$ can explain the high rate of diffractive events. Diffractive reactions may therefore be well suited to search for saturation effects in the proton structure when $x$ reaches sufficiently small values [16].

Several theoretical QCD approaches have been proposed to interpret the dynamics of diffractive DIS. A general theoretical framework is provided by the QCD collinear factorisation theorem for semi-inclusive DIS cross sections such as that for $e p \rightarrow e X p[17,18]$. This implies that the concept of diffractive parton distribution functions (DPDFs) may be introduced, representing conditional proton parton probability distributions under the constraint of a leading final state proton with a particular four-momentum. Empirically, an additional factorisation has been found to apply to good approximation, whereby the variables which describe the proton vertex factorise from those describing the hard interaction (proton vertex factorisation) $[19,20]$. The dependence of the DPDFs on the kinematic variables related to the proton vertex can be parametrised conveniently using Regge formalism, which amounts to a description of diffraction in terms of the exchange of a factorisable Pomeron $(\mathbb{P})[21,22]$ with universal parton densities. Several authors have analysed diffractive DIS data to extract DPDFs $[4,8$, 10, 23-34], with the conclusion that the data are compatible with proton vertex factorisation at low fractional proton energy losses, $x_{\mathbb{P}}$, and for photon virtualities $Q^{2}$ above $\sim 5 \mathrm{GeV}^{2}$. The DPDFs extracted in these publications consistently find a dominant gluon contribution. At larger $x_{\mathbb{P}}$

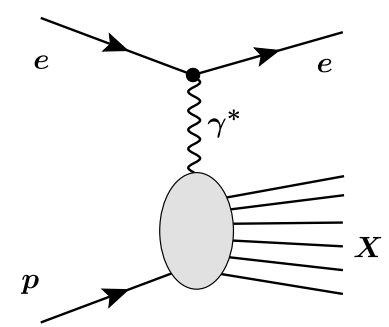

(a)

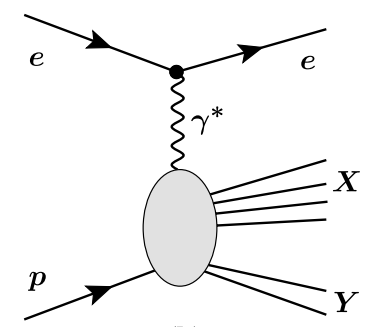

(b)
Fig. 1 Inclusive (a) and diffractive (b) deep inelastic scattering

$\left(x_{\mathbb{P}}>0.1\right)$, a separately factorisable sub-leading Reggeon exchange $(\mathbb{R})$, with a different $x_{\mathbb{P}}$ dependence and partonic composition, is usually included to maintain a good description.

The diffractive cross section can also be interpreted within the dipole model. In this picture, the virtual photon fluctuates into a colour singlet $q \bar{q}$ pair (or dipole) of transverse size $r \sim 1 / Q$, which subsequently undergoes a hard scattering with the proton [35-40]. In the low $\beta$ domain, it is expected that $q \bar{q}-g$ dipoles also contribute to inclusive diffraction [41]. In a recent unified saturation description of diffractive DIS good agreement with data has been obtained in the full $Q^{2}$ range down to $\sim 3 \mathrm{GeV}^{2}$ [16]. This dipole model uses the parametrisation for the dipole scattering amplitude obtained in [42], which is an extension of the saturation model presented in [37] containing in addition heavyquark contributions. This approach is interesting because it relates the diffractive process, in the regime $x_{\mathbb{P}}<0.01$ in which saturation is expected to be relevant, to the DIS inclusive process. The description of the diffractive process is obtained without extra parameter by considering the dipole cross section $\sigma_{0}$ and the diffractive slope $B_{D}$ being directly related.

In this paper, a new measurement of the diffractive neutral current DIS cross section is presented. This is based upon $\mathrm{H} 1$ data for which there is an absence of hadronic activity in a large rapidity region extending close to the outgoing proton beam direction. The data were recorded with the H1 detector in the years 1999-2000 and 2004-2007, when HERA collided protons of $920 \mathrm{GeV}$ energy with $27.6 \mathrm{GeV}$ electrons and positrons. The analysed data cover the low and medium $Q^{2}$ region from 3 to $105 \mathrm{GeV}^{2}$. A combination with previous measurements obtained by $\mathrm{H} 1$, also using Large Rapidity Gap (LRG) events and based on low and medium $Q^{2}$ data from 1997 and high $Q^{2}$ data from 19992000 [10], is performed in order to provide a single set of diffractive cross sections for $Q^{2}$ up to $1600 \mathrm{GeV}^{2}$. The results are compared with QCD calculations based on DPDFs extracted from previous $\mathrm{H} 1$ data [10] and with recent dipole model predictions [16]. 


\section{Diffractive DIS kinematics variables and observables}

The kinematics of the inclusive DIS process can be described by the Lorentz invariants

$x=\frac{-q^{2}}{2 P \cdot q}, \quad y=\frac{P \cdot q}{P \cdot k}, \quad Q^{2}=-q^{2}$,

where $P$ and $k$ are the 4-momenta of the incident proton and electron ${ }^{1}$ respectively and $q$ is the 4-momentum of the exchanged virtual photon. The kinematics of the diffractive process can be described in addition by the invariant masses $M_{X}$ and $M_{Y}$ of the systems $X$ and $Y$, and

$t=\left(P-P_{Y}\right)^{2}$,

$\beta=\frac{-q^{2}}{2 q \cdot\left(P-P_{Y}\right)}=\frac{Q^{2}}{Q^{2}+M_{X}^{2}-t}$,

$x_{\mathbb{P}}=\frac{q \cdot\left(P-P_{Y}\right)}{q \cdot P}=\frac{Q^{2}+M_{X}^{2}-t}{Q^{2}+W^{2}-m_{P}^{2}}=\frac{x}{\beta}$,

where $P_{Y}$ is the 4-momentum of system $Y, W^{2}=(q+P)^{2}$ is the squared centre of mass energy of the virtual photonproton system and $m_{P}$ is the proton mass. The variable $x_{\mathbb{P}}$ is the fractional momentum loss of the incident proton. The quantity $\beta$ has the form of a Bjorken variable defined with respect to the momentum $P-P_{Y}$ lost by the initial proton.

In analogy to the inclusive DIS cross section, the inclusive diffractive cross section integrated over $t$ for $e p \rightarrow e X Y$ in the one-photon exchange approximation can be written in terms of diffractive structure functions $F_{2}^{D(3)}$ and $F_{L}^{D(3)}$ as

$$
\begin{aligned}
\frac{\mathrm{d}^{3} \sigma^{e p \rightarrow e X Y}}{\mathrm{~d} Q^{2} \mathrm{~d} \beta \mathrm{d} x_{\mathbb{P}}}= & \frac{4 \pi \alpha_{\mathrm{em}}^{2}}{\beta Q^{4}}\left[\left(1-y+\frac{y^{2}}{2}\right) F_{2}^{D(3)}\left(\beta, Q^{2}, x_{\mathbb{P}}\right)\right. \\
& \left.-\frac{y^{2}}{2} F_{L}^{D(3)}\left(\beta, Q^{2}, x_{\mathbb{P}}\right)\right],
\end{aligned}
$$

where $\alpha_{\mathrm{em}}=1 / 137$. The structure function $F_{L}^{D(3)}$ corresponds to longitudinal polarisation of the virtual photon. The reduced diffractive cross section is defined by

$$
\begin{aligned}
\sigma_{r}^{D(3)}\left(Q^{2}, \beta, x_{\mathbb{P}}\right) & =\frac{\beta Q^{4}}{4 \pi \alpha_{\mathrm{em}}^{2}} \frac{1}{\left(1-y+\frac{y^{2}}{2}\right)} \frac{\mathrm{d}^{3} \sigma^{e p \rightarrow e X Y}}{\mathrm{~d} Q^{2} \mathrm{~d} \beta \mathrm{d} x_{\mathbb{P}}} \\
& =F_{2}^{D(3)}-\frac{y^{2}}{1+(1-y)^{2}} F_{L}^{D(3)} .
\end{aligned}
$$

\section{Experimental procedure}

\section{$3.1 \mathrm{H} 1$ detector}

A detailed description of the $\mathrm{H} 1$ detector can be found elsewhere [43-45]. Here, only the detector components relevant for the present analysis are briefly described. H1 uses

\footnotetext{
${ }^{1}$ In this paper the term "electron" is used generically to refer to both electrons and positrons.
}

a right-handed coordinate system with the $z$ axis along the beam direction and the $+z$ or "forward" direction being that of the outgoing proton beam. The polar angle $\theta$ is defined with respect to the $z$ axis and the pseudorapidity is given by $\eta=-\ln \tan \theta / 2$.

The liquid argon (LAr) calorimeter $\left(4^{\circ} \leq \theta \leq 154^{\circ}\right)$ is situated inside a solenoidal magnet. The energy resolutions for electromagnetic and hadronic showers are $\sigma(E) / E \simeq$ $11 \% / \sqrt{E / \mathrm{GeV}} \oplus 1 \%$ and $\sigma(E) / E \simeq 50 \% / \sqrt{E / \mathrm{GeV}} \oplus$ $2 \%$, respectively, as obtained from test beam measurements [46, 47]. The backward region $\left(153^{\circ}<\theta<176^{\circ}\right)$ is covered by a lead scintillating fibre calorimeter, the SpaCal [45], which has both electromagnetic and hadronic sections. Its energy resolution for electromagnetic showers is $\sigma(E) / E \simeq 7.1 \% / \sqrt{E / \mathrm{GeV}} \oplus 1 \%$. A tracking chamber placed in front of the SpaCal, the backward drift chamber (BDC) for the period 1999-2000 and the backward proportional chamber (BPC) for the period 2004-2007, is used to identify the scattered electron and to determine its position.

The main component of the central tracking detector is the central jet chamber $\operatorname{CJC}\left(20^{\circ}<\theta<160^{\circ}\right)$ which consists of two coaxial cylindrical drift chambers with wires parallel to the beam direction. The measurement of charged particle transverse momenta is performed in a magnetic field of $1.16 \mathrm{~T}$, which is uniform over the full tracker volume. The forward tracking detector, $\left(\theta<30^{\circ}\right)$ is used to determine the vertex position for events where no CJC track is reconstructed.

The forward components of the $\mathrm{H} 1$ detector, used here to tag hadronic activity at large pseudorapidity $(3.5<\eta<7)$, are the Plug forward calorimeter, the forward muon detector (FMD), the proton remnant tagger (PRT) and the forward tagging system (FTS). The Plug enables energy measurements to be made in the pseudorapidity range $3.5<\eta<5.5$. It is positioned around the beam-pipe at $z=4.9 \mathrm{~m}$. The FMD consists of a series of drift chambers covering the range $1.9<\eta<3.7$. Primary particles produced at larger $\eta$ can be detected indirectly in the FMD if they undergo a secondary scattering with the beam pipe or other adjacent material. For the period 1999-2000, secondary particles, or the scattered proton at very high $|t|$, can also be detected by the PRT, covering the range $6.5<\eta<7.5$, which is located at $24 \mathrm{~m}$ from the interaction point and consists of layers of scintillator surrounding the beam pipe. In the period 2004-2007, the PRT is replaced by the FTS which consists of four stations of scintillators arranged around the proton beam pipe at $z=26 \mathrm{~m}, z=28 \mathrm{~m}, z=53 \mathrm{~m}$ and $z=92 \mathrm{~m}$. Only the stations at $26 \mathrm{~m}$ and $28 \mathrm{~m}$ are used to tag proton dissociation, since further downstream elastically scattered protons often hit the beam-pipe.

The luminosity is determined from the rate of BetheHeitler processes measured using a calorimeter located close to the beam pipe at $z=-103 \mathrm{~m}$ in the backward direction. 


\subsection{Data samples}

Different event samples corresponding to different $Q^{2}$ ranges are analysed in this paper. For the interval $3 \leq Q^{2} \leq$ $25 \mathrm{GeV}^{2}$, a 'minimum bias' (MB) sample corresponding to an integrated luminosity of $3.5 \mathrm{pb}^{-1}$ is used, which was recorded during a special data taking period in 1999 with dedicated low $Q^{2}$ electron triggers. For photon virtualities in the interval $10 \leq Q^{2} \leq 105 \mathrm{GeV}^{2}$, data taken throughout the periods 1999-2000 and 2004-2007 are used, corresponding to a total integrated luminosity of $371 \mathrm{pb}^{-1}$. These cross section measurements are combined with previously published H1 LRG data [10]. All event samples are summarised in Table 1.

\subsection{Event selection and kinematic reconstruction}

DIS events are selected by requiring a localised energy deposit (cluster) in the SpaCal calorimeter with an energy greater than $10 \mathrm{GeV}$, ensuring a trigger efficiency close to $100 \%$. The cluster radius of the electron candidate is required to be less than $4 \mathrm{~cm}$, as expected for an electromagnetic shower. In order to avoid losses of energy into the beam-pipe, the radial distance between the beam axis and the cluster barycentre is required to be larger than $11 \mathrm{~cm}$. For the data recorded in 1999-2000, a track segment was required in the $\mathrm{BDC}$, matching the cluster in the SpaCal calorimeter within $3 \mathrm{~cm}$.

Cosmic ray and beam induced backgrounds are reduced to negligible levels by requiring a vertex reconstructed within $35 \mathrm{~cm}$ of the nominal interaction point and the timing of the signals from the tracking detector to be within the interval expected for $e p$ collisions. Radiative events and photoproduction events in which a hadron is misidentified as the scattered electron are suppressed by requiring $\sum_{i}\left(E^{i}-p_{z}^{i}\right)>37 \mathrm{GeV}$, where $E^{i}$ and $p_{z}^{i}$ are the energy and longitudinal momentum of all detected particles $i$, including the scattered electron.

Table 1 Summary of the data samples used in the analysis

\begin{tabular}{llll}
\hline Data Set & $Q^{2}$ range & Proton Energy & Luminosity \\
& $\left(\mathrm{GeV}^{2}\right)$ & $E_{p}(\mathrm{GeV})$ & $\left(\mathrm{pb}^{-1}\right)$
\end{tabular}

New data samples

\begin{tabular}{|c|c|c|c|}
\hline 1999 MB & $3<Q^{2}<25$ & 920 & 3.5 \\
\hline 1999-2000 & $10<Q^{2}<105$ & 920 & 34.3 \\
\hline 2004-2007 & $10<Q^{2}<105$ & 920 & 336.6 \\
\hline \multicolumn{4}{|c|}{ Previously published data samples } \\
\hline $1997 \mathrm{MB}$ & $3<Q^{2}<13.5$ & 820 & 2.0 \\
\hline 1997 & $13.5<Q^{2}<105$ & 820 & 10.6 \\
\hline 1999-2000 & $133<Q^{2}<1600$ & 920 & 61.6 \\
\hline
\end{tabular}

The inclusive DIS kinematic variables, $x, Q^{2}$ and the inelasticity $y$, are reconstructed using the techniques introduced in [4]. In order to optimise the resolution throughout the measured $y$ range, information is exploited from both the scattered electron and the hadronic final state according to

$$
y=y_{d}+\left(y_{e}^{2}-y_{d}^{2}\right), \quad Q^{2}=\frac{4 E_{e}^{2}(1-y)}{\tan ^{2}\left(\theta_{e}^{\prime} / 2\right)}, \quad x=\frac{Q^{2}}{s y} .
$$

Here, $y_{e}$ and $y_{d}$ denote the values of $y$ obtained from the scattered electron only ('electron method') and from the angles of the electron and the hadronic final state ('double angle method'), respectively [10]. $E_{e}$ is the electron beam energy and $\theta_{e}^{\prime}$ is the polar angle of the scattered electron. In order to ensure a reasonable containment of the hadronic final state in the central detectors only events with $y>0.04$ are selected.

A sub-sample of events where a diffractive exchange dominates is selected by requiring that no signal is recorded above noise levels in a number of forward components of the $\mathrm{H} 1$ detector. The pseudorapidity $\eta_{\max }$ of the most forward energy deposit in the LAr calorimeter above a noise threshold of $800 \mathrm{MeV}$ is required to be less than 3.3. At most one hit pair should be present in the first two layers of the FMD. The energy measured in the Plug calorimeter is required to be smaller than $7 \mathrm{GeV}$. For the period 1999-2000, it is required that there is no signal in the first five layers of the PRT. For the period 2004-2007, it is required that there are no hits in the $26 \mathrm{~m}$ and $28 \mathrm{~m}$ stations of the FTS. After these selection criteria are applied, the systems $X$ and $Y$ are well separated by an LRG. The system $X$ is fully contained in the main part of the $\mathrm{H} 1$ detector and the system $Y$ goes unobserved into the beam pipe.

The large rapidity gap selection yields a sample which is dominated by the elastic ${ }^{2}$ process $e p \rightarrow e X p$, with the outgoing proton transverse momentum $p_{t, p}$, and hence $|t| \simeq$ $p_{t, p}^{2}$, being relatively small. However, there is an admixture of proton dissociative events, $e p \rightarrow e X Y$, where the proton dissociation system has a small mass $M_{Y}$. The ranges of sensitivity of the measurement in $M_{Y}$ and $t$ are determined by the acceptances of the forward detectors which are used to identify the large rapidity gap. In order to keep the uncertainties arising from proton dissociation small and to ease comparisons with previous data [10], the measurement is integrated over the region $M_{Y}<1.6 \mathrm{GeV},|t|<1 \mathrm{GeV}^{2}$. The correction factors applied to account for the net migrations about these limits are determined by evaluation of the forward detector response to elastic proton and proton dissociative processes, using the Monte Carlo program DIFFVM [48]. This correction is $9 \%$ for the $1999 \mathrm{MB}$ and

\footnotetext{
${ }^{2}$ Here the term "elastic" is used to refer to the process $e p \rightarrow e X Y$ with $Y=p$ and not to $e p \rightarrow e p$.
} 
1999-2000 samples and $13 \%$ in 2004-2007. Noise in the forward detector components results in some events being wrongly rejected from the samples. These losses are determined using randomly triggered events which are overlaid with simulated events.

The reconstruction of hadrons combines information from the calorimeters and vertex-fitted tracks in the central tracker without double counting $[49,50]$. The reconstructed hadronic final state four vector $P_{H}$ is then defined as the vector sum of all reconstructed hadrons. The invariant mass $M_{X}$ of the final state system $X$ is obtained by

$M_{X}=\sqrt{P_{H}^{2} \frac{y}{y_{h}}}$,

with $y_{h}=\sum_{h}\left(E^{h}-p_{z}^{h}\right) / 2 E_{e}$, where the sum runs over all reconstructed hadrons. The factor $y / y_{h}$ is included to improve the resolution at large $y$, where losses in the backward direction become large. The kinematic reconstruction method used here leads to a resolution in $M_{X}$ varying from 13 to $22 \%$ in the measured kinematic range. In this analysis, $M_{X}$ is required to be above $1 \mathrm{GeV}$. According to Eq. (2) and neglecting $t$, the diffractive variables $\beta$ and $x_{\mathbb{P}}$ are obtained from:

$\beta=\frac{Q^{2}}{Q^{2}+M_{X}^{2}} ; \quad x_{\mathbb{P}}=\frac{x}{\beta}$.

\subsection{Monte Carlo simulations}

Corrections for detector inefficiencies and acceptance losses due to the event selection cuts are evaluated bin-by-bin directly from the data or by using a Monte Carlo (MC) simulation of the detectors. Corrections for migrations in the kinematic variables due to the finite resolution are determined using MC programs. All generated MC events are passed through a detailed, GEANT [51] based, simulation of the H1 detector, which takes into account the running conditions of the different data taking periods, and are subject to the same reconstruction and analysis chain as used for data.

Diffractive DIS is modelled using the RAPGAP Monte Carlo generator [52]. The RAPGAP event generator implements the exchange of a partonic Pomeron or meson with leading order QCD matrix elements. The Pomeron and meson fluxes and the parton distributions used in the event simulation are based on the DPDF fit to previous H1 data (H1 2006 DPDF Fit B) [10]. At low $Q^{2}$, H1 2006 DPDF Fit B undershoots the data, as observed previously [10]. For $Q^{2}<7 \mathrm{GeV}^{2}$, RAPGAP is therefore reweighted by a parametrisation, depending on $Q^{2}$ and $\beta$, to describe the present data. Higher order QCD radiation is modelled using initial and final state parton showers in the approximation of leading logarithms [53]. Hadronisation is simulated using the Lund string model [54] as implemented in JETSET [55]. QED radiative effects, including virtual loop corrections, are taken into account via an interface to the HERACLES program [56]. Migrations into the sample from the region $M_{Y}>5 \mathrm{GeV}$ are studied by using RAPGAP in the inclusive DIS mode. At low $M_{X}$, where the presence of the meson resonances $\rho, \omega, \phi$ becomes important, the DIFFVM MC [48] is used in addition. The Monte Carlo program COMPTON [57] is used to simulate single dissociation and inelastic Bethe-Heitler events.

Background from $e p$ interactions may arise from photoproduction events $\left(Q^{2} \sim 0\right)$ in which the scattered lepton signal is faked by a hadron detected in the SpaCal calorimeter. It is estimated using the PHOJET Monte Carlo model [58] and found to be negligible in this analysis. Other backgrounds, such as those due to interactions of the beams with the remaining gas in the beam pipe or with beam line elements upstream of the $\mathrm{H} 1$ detector, are also found to be negligible.

\subsection{Systematic uncertainties}

A detailed systematic error analysis has been performed, in which the sensitivity of the measurements to variations in the efficiencies and energy scales of the detector components and to the details of the correction procedure is tested. The systematic error sources leading to uncertainties which are correlated between data points are determined from the agreement of the simulation with data in this analysis and are listed below.

- The uncertainty on the SpaCal electromagnetic energy scale is evaluated to be $0.5 \%$ and $0.4 \%$ for $1999-2000$ and 2004-2007 data, respectively. The uncertainties in the relative alignment of the different detector components are reflected in possible biases in the electron polar angle measurement at the level of $0.5 \mathrm{mrad}$ and $1 \mathrm{mrad}$ for 1999-2000 and 2004-2007 data, respectively.

- The hadronic energy scale of the LAr calorimeter is known to $2 \%$ for the $1999 \mathrm{MB}$ sample and to $1.5 \%$ for all other samples.

- Imperfect treatment of calorimeter noise can result in a bias in the reconstruction of $M_{X}$. The corresponding uncertainty is evaluated by varying the amount of calorimeter energy classified as noise by $10 \%$. This level of precision is determined by comparing the calorimeter noise subtracted in the data with that in the Monte Carlo model, which includes a simulation of noise based on randomly triggered events.

- The efficiency with which the FMD registers activity when there is hadronic energy flow in its acceptance region is varied in the simulation by $5 \%$ for $1999-2000$ and $4 \%$ for 2004-2007. For the PRT and FTS, this efficiency is varied by $20 \%$ and $7 \%$, respectively. The Plug energy 
scale is varied by $10 \%$. These levels of uncertainty are obtained by comparison of the present data with the Monte Carlo simulation for samples in which forward detector activity is required.

- The model dependences of the acceptance and migration corrections and of the background subtractions are estimated by varying the details of the Monte Carlo simulation within the limits permitted by the present data. In the RAPGAP simulation of diffraction, the $x_{\mathbb{P}}$ distribution is reweighted by $\left(1 / x_{\mathbb{P}}\right)^{ \pm 0.05}$, the $\beta$ distribution by $\beta^{ \pm 0.05}$ and $(1-\beta)^{ \pm 0.05}$, the $t$ distribution by $e^{ \pm t}$ [12] and the $Q^{2}$ distribution by $\left(\log Q^{2}\right)^{ \pm 0.2}$. The reweighting in $t$ and $(1-\beta)$ are found to have a negligible effect on the measured cross sections. For $Q^{2}<7 \mathrm{GeV}^{2}$, an additional uncertainty on the shape of the $\beta$ distribution is introduced to account for the poor description of the data by RAPGAP in this phase space region. This results in an additional uncertainty below $1 \%$ on the measured cross sections. The normalisation of the sub-leading meson exchange in RAPGAP is varied by $\pm 25 \%$ and that of the vector meson production simulation (DIFFVM) is varied by $\pm 50 \%$. The uncertainty in the background from high $M_{Y}$, as simulated by the inclusive RAPGAP MC, is taken to be $100 \%$. These normalisation uncertainties are determined in this analysis by comparing MC predictions to data in alternative event selections.

- The model dependence of the bin centre corrections is estimated by comparing the results obtained using the $\mathrm{H} 1$ 2006 DPDF Fit A and Fit B sets. It results in a sizeable correlated uncertainty of up to $3 \%$ only at the largest $\beta$ values.

Several further uncertainties, listed below, affect all data points in an identical manner and are thus considered as normalisation uncertainties.

- The uncertainty on the factor correcting the measured cross section to the kinematic range $M_{Y}<1.6 \mathrm{GeV}$, $|t|<1 \mathrm{GeV}^{2}$ is $7 \%$ (see Sect. 3.3). The dominant contribution to this uncertainty arises from variations in the assumed ratio of proton dissociation to elastic proton cross sections in the range 0.5 to 2.0. Fluctuations of the noise level in the forward detector components are also taken into account.

- The normalisation uncertainty arising from the luminosity measurement is $1.5 \%$ for the $1999 \mathrm{MB}$ and $1999-2000$ data samples and 3.5\% for 2004-2007 data.

A third class of systematic errors leads to uncertainties which are considered not to be correlated between data points.

- The calculated acceptance of the $\eta_{\max }$ cut depends on the modelling of the hadronic final state topology. The associated uncertainty is estimated from the effect of using an alternative model for higher order QCD processes (the colour dipole approach [59] as implemented in ARIADNE [60] in place of parton showers). This results in an uncertainty which depends to good approximation on $x_{\mathbb{P}}$ only and varies between $1.2 \%$ at $x_{\mathbb{P}}=0.0003$ and $4 \%$ at $x_{\mathbb{P}}=0.01$.

- The uncertainty on the trigger efficiency is $1 \%$, as determined in this analysis.

- The uncertainty on radiative corrections is $1 \%$.

The total systematic uncertainty on each data point is formed by adding the individual contributions in quadrature. A full decomposition of the systematic errors on the measured cross sections is available elsewhere [61]. Away from the boundaries of the kinematic region, the systematic error excluding the normalisation uncertainty ranges from $3 \%$ to $9 \%$ (4\% to $10 \%$ for $1999 \mathrm{MB}$ data), with no single source of uncertainty dominating. These systematic uncertainties are to be compared with statistical errors of the order of $1 \%$ in the intermediate $Q^{2}$ domain (1999-2000 and 2004-2007 data) and $5 \%$ for the low $Q^{2}$ region (1999 MB data). The overall normalisation uncertainties for each data set are of the order of 7 to $8 \%$.

\section{Results and discussion}

\subsection{Diffractive cross section measurements and combination}

The 1999 MB, 1999-2000 and 2004-2007 data samples are used to measure the reduced diffractive cross section $\sigma_{r}{ }^{D(3)}\left(Q^{2}, \beta, x_{\mathbb{P}}\right)$. The bins in $Q^{2}, \beta$ and $x_{\mathbb{P}}$ are chosen to have a width always larger than twice the experimental resolution. The cross section measurements are corrected to fixed values of $Q^{2}, \beta$ and $x_{\mathbb{P}}$ for each bin using predictions from the H1 2006 DPDF Fit B. These corrections are of the order of $5 \%$ in average. The details of this procedure including bin definitions are the same as for the previous $\mathrm{H} 1$ measurement [10]. The measurements are quoted at the Born level after correcting for QED radiative effects. Radiative corrections are calculated bin by bin using the HERACLES program [56] interfaced to RAPGAP. They are smaller than $5 \%$ for all measured data points. The results are corrected to the region $M_{Y}<1.6 \mathrm{GeV}$, and $|t| \leq 1 \mathrm{GeV}^{2}$.

The new data sets of this analysis are combined with the previously published $\mathrm{H} 1$ measurements from the 1997 data [10] using the $\chi^{2}$ minimisation method developed for the combination of inclusive DIS cross sections [62-64]. In the year 1997, the data were taken at a centre-of-mass energy of $\sqrt{s}=300 \mathrm{GeV}$ whilst all the other data samples were taken at $\sqrt{s}=319 \mathrm{GeV}$. The 1997 measurements are therefore corrected to $\sqrt{s}=319 \mathrm{GeV}$ using H1 2006 DPDF Fit B to parametrise $F_{L}^{D(3)}$. This correction is always below $1 \%$ in the kinematic domain covered. The error associated to 
this correction is estimated by varying the $F_{L}^{D(3)}$ prediction from H1 2006 DPDF Fit B by $\pm 100 \%$, which is conservative with respect to the direct measurement of $F_{L}^{D(3)}$ [15]. The combined cross section measurements are given for $\sqrt{s}=319 \mathrm{GeV}$. For $x_{\mathbb{P}}=0.03$ and for $Q^{2}>133 \mathrm{GeV}^{2}$ in all $x_{\mathbb{P}}$ bins, only cross section values measured previously [10] are available.

The combination is performed taking into account correlated systematic uncertainties. Systematic uncertainties associated with detector modelling are treated as uncorrelated between data sets. Model systematic uncertainties on the acceptance and migration corrections are considered to be completely correlated between data sets. An overall normalisation uncertainty of $4 \%$ is also considered as correlated between data sets. It corresponds to the fraction of the correction factor accounting for smearing about the $M_{Y}$ and $t$ boundaries (see Sect. 3.3), whose determination method is common to all data sets. There are 597 data points averaged to 277 cross section measurements. The data from the different running periods show a reasonable consistency, with the total $\chi^{2}$ per degree of freedom $\left(n_{\text {dof }}\right)$ of $\chi^{2} / n_{\text {dof }}=371 / 320$. The adjustments of the relative normalisations are small, with the normalisation of the $1999 \mathrm{MB}$ data set staying constant and the other data samples shifting by at most $1.3 \%$. The distribution of pulls [64] of each data point relative to the combined cross section measurements is shown in Fig. 2 and does not exhibit large tensions. The largest deviations are observed in the lowest $Q^{2}$ bins at $x_{\mathbb{P}}=0.01$.

The $\beta$ dependence of the combined reduced cross section measurements, multiplied by $x_{\mathbb{P}}$, is shown in Figs. 3, 4 , 5, 6 for fixed values of $x_{\mathbb{P}}=0.0003,0.001,0.003$ and 0.01 and is compared with the previously published cross sec- tion measurements [10] and with the prediction from the $\mathrm{H} 1$ 2006 DPDF Fit B. The $Q^{2}$ dependence is presented in Fig. 7. A significant reduction of statistical errors is observed. The new combined data have a total uncertainty between $4 \%$ and $7 \%$ whereas they were typically of the order of $7 \%$ and $10 \%$ in the previously published results.

For $x_{\mathbb{P}}=0.03$ only the previous measurements [10] exist. They are only slightly modified by the combination procedure. The resulting $\beta$ and $Q^{2}$ dependences are shown in Fig. 8. The results for all $x_{\mathbb{P}}$ bins are also provided in numerical form in Tables 2 to 7 and in [61]. Statistical together with uncorrelated and point-to-point correlated systematic uncertainties are shown.

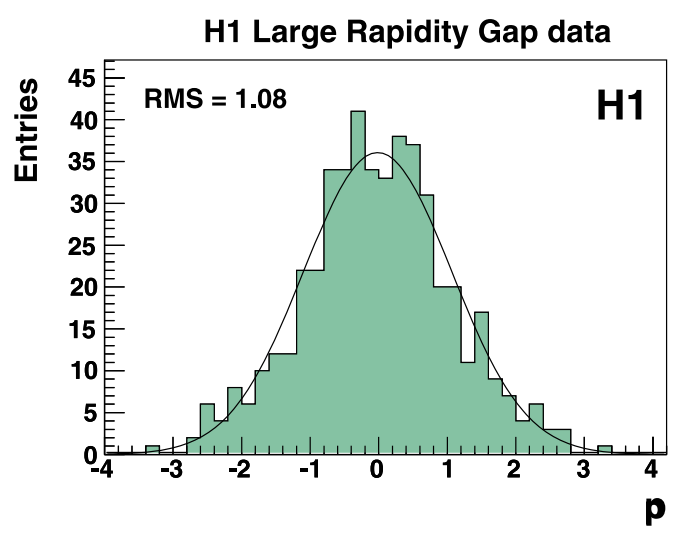

Fig. 2 Distribution of pulls $p$ for all data samples. There are no entries outside the histogram range. The RMS gives the root mean square of the distribution calculated as $\overline{p^{2}}$. The curve shows the result of a binned log-likelihood Gaussian fit to the distribution

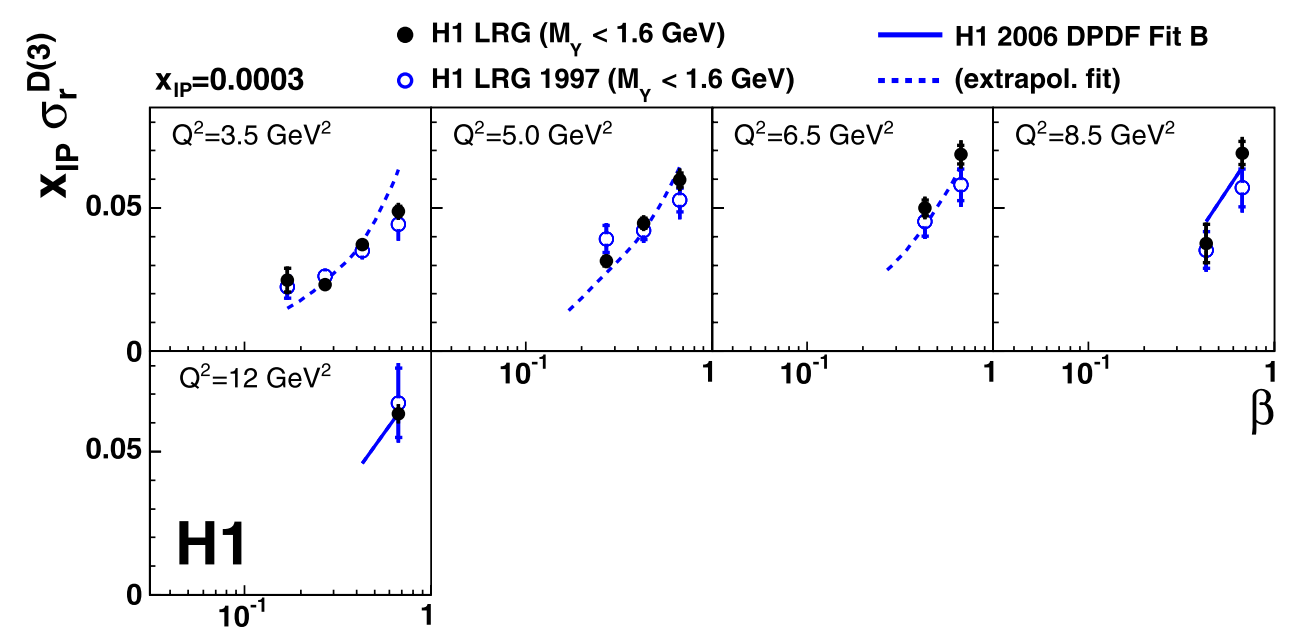

Fig. 3 The $\beta$ dependence of the reduced diffractive cross section, multiplied by $x_{\mathbb{P}}$, at a fixed value of $x_{\mathbb{P}}=0.0003$, resulting from the combination of all data samples. Previously published H1 measurements [10] are also displayed as open points. The inner and outer error bars on the data points represent the statistical and total uncertainties, respectively.
Overall normalisation uncertainties of $4 \%$ and $6.2 \%$ on the combined and previous data, respectively, are not shown. Predictions from the $\mathrm{H} 1$ 2006 DPDF Fit B [10] are represented by a curve in kinematic regions used to determine the DPDFs and by a dashed line in regions which were excluded from the fit (see Sect. 4.3) 
Fig. 4 The $\beta$ dependence of the reduced diffractive cross section, multiplied by $x_{\mathbb{P}}$, at a fixed value of $x_{\mathbb{P}}=0.001$, resulting from the combination of all data samples. Details are explained in the caption of Fig. 3

Fig. 5 The $\beta$ dependence of the reduced diffractive cross section, multiplied by $x_{\mathbb{P}}$, at a fixed value of $x_{\mathbb{P}}=0.003$, resulting from the combination of all data samples. Details are explained in the caption of Fig. 3
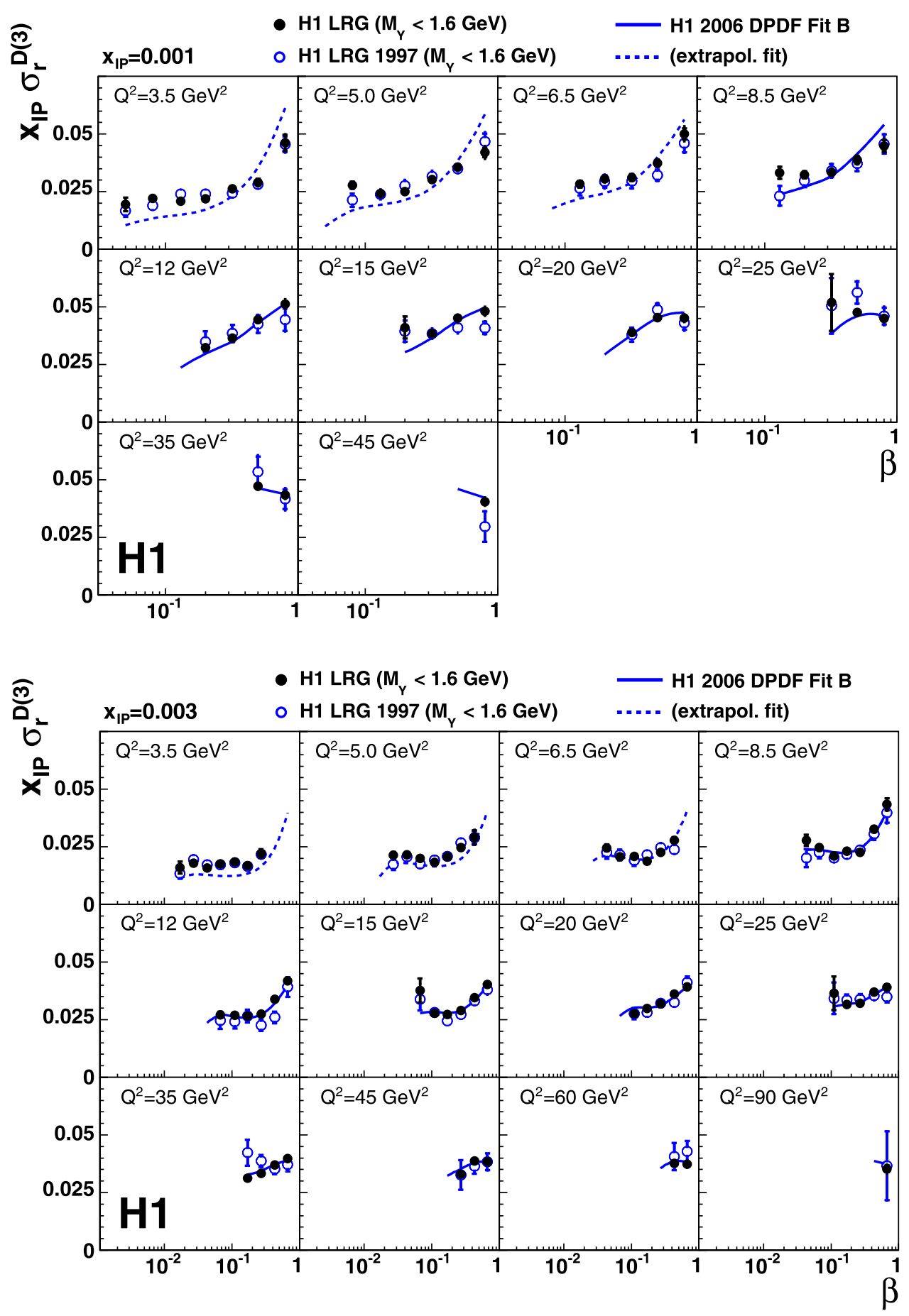

\subsection{Comparisons with other measurements}

The combined reduced cross section $\sigma_{r}{ }^{D(3)}$ can be compared with other $\mathrm{H} 1$ measurements obtained by a direct measurement of the outgoing proton using the H1 Forward Proton Spectrometer (FPS) [12]. The cross section $e p \rightarrow e X Y$ measured here with the LRG data includes proton dissociation to any system $Y$ with a mass in the range $M_{Y}<1.6 \mathrm{GeV}$, whereas in the cross section measured with the FPS the sys- tem $Y$ is defined to be a proton. The FPS results are interpolated to the $Q^{2}, \beta$ and $x_{\mathbb{P}}$ bin centre values of the LRG data using a parametrisation of the H1 2006 DPDF Fit B. Only FPS data with interpolation corrections between 0.8 and 1.25 are used. The ratio of the two measurements is then formed for each $\left(Q^{2}, \beta, x_{\mathbb{P}}\right)$ point for $x_{\mathbb{P}}=0.01$ and $x_{\mathbb{P}}=0.03$, at which both LRG and FPS data are available. The FPS data set covers only the 2004-2007 running period whilst the LRG data also includes cross sections from 1997 , 
Fig. 6 The $\beta$ dependence of the reduced diffractive cross section, multiplied by $x_{\mathbb{P}}$, at a fixed value of $x_{\mathbb{P}}=0.01$, resulting from the combination of all data samples. Details are explained in the caption of Fig. 3

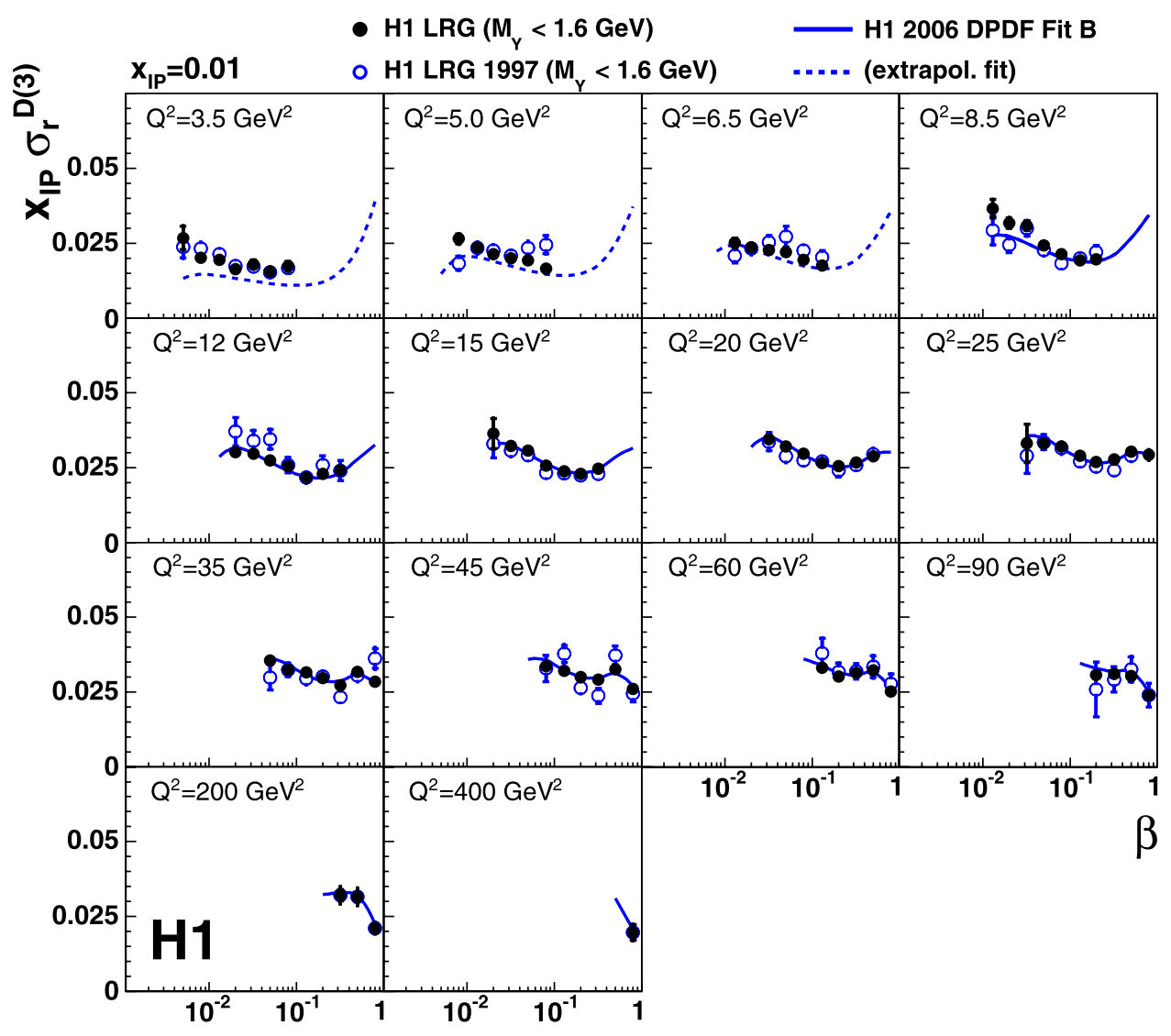

$1999 \mathrm{MB}$ and 1999-2000 running periods, for $x_{\mathbb{P}}=0.01$ and only 1997 data for $x_{\mathbb{P}}=0.03$. The two LRG and FPS data sets are therefore statistically independent to a large extent. As also the dominant sources of systematic errors in the two data sets are different, correlations between the uncertainties on the FPS and LRG data are neglected. The global weighted average of the cross section ratio LRG/FPS is

$$
\begin{aligned}
\frac{\sigma\left(M_{Y}<1.6 \mathrm{GeV}\right)}{\sigma(Y=p)}= & 1.203 \pm 0.019 \text { (exp.) } \\
& \pm 0.087 \text { (norm.) }
\end{aligned}
$$

where the experimental uncertainty is a combination of statistical and uncorrelated systematic uncertainties on the measurements. In Fig. 9 the combined LRG cross section measurements as a function of $Q^{2}$ are compared with the interpolated FPS data rescaled by a factor 1.2, following the above determination. A good agreement between the two measurements is observed.

The combined H1 LRG cross section are also compared with the most recent measurements by the ZEUS experiment using a similar LRG selection [14]. These ZEUS diffractive data have been determined for identical $\beta$ and $x_{\mathbb{P}}$ values, but at different $Q^{2}$ values to H1. In order to match the $M_{Y}<1.6 \mathrm{GeV}$ range of the $\mathrm{H} 1$ data, a global factor of $0.91 \pm 0.07$ [14] is applied to the ZEUS LRG data. The comparison for $M_{Y}<1.6 \mathrm{GeV}$ between the $\mathrm{H} 1$ data and the rescaled ZEUS data is shown in Fig. 10. The ZEUS data tend to remain higher than those of $\mathrm{H} 1$ by $\sim 10 \%$ on average. This difference in normalisation is consistent with the $8 \%$ uncertainty on the proton-dissociation correction factor of $0.91 \pm 0.07$ applied to ZEUS data combined with the normalisation uncertainties of the two data sets of $4 \%$ (H1) and $2.25 \%$ (ZEUS). This normalisation difference is also similar to that of $0.85 \pm 0.01$ (stat.) \pm 0.03 (sys.) ${ }_{-0.12}^{+0.09}$ (norm.) between the H1 FPS and the ZEUS LPS tagged-proton data sets [12]. Deviations are observed between the $\beta$ dependences of the two measurements at the highest and lowest $\beta$ values. However a good agreement of the $Q^{2}$ dependence is observed throughout most of the phase space.

\subsection{Comparison with models}

Figures 3 to 10 show the measurements compared to predictions based on the H1 2006 DPDF Fit B. The DPDF fit assuming proton vertex factorisation used in the previous $\mathrm{H} 1$ analysis [10] became unstable when data points with $Q^{2}<8.5 \mathrm{GeV}^{2}$ were included. Therefore, only an extrapolation of the DPDFs predictions to this kinematic domain is indicated as dashed lines in these figures. In Fig. 10 the data are compared also with predictions of the dipole model [16]. 

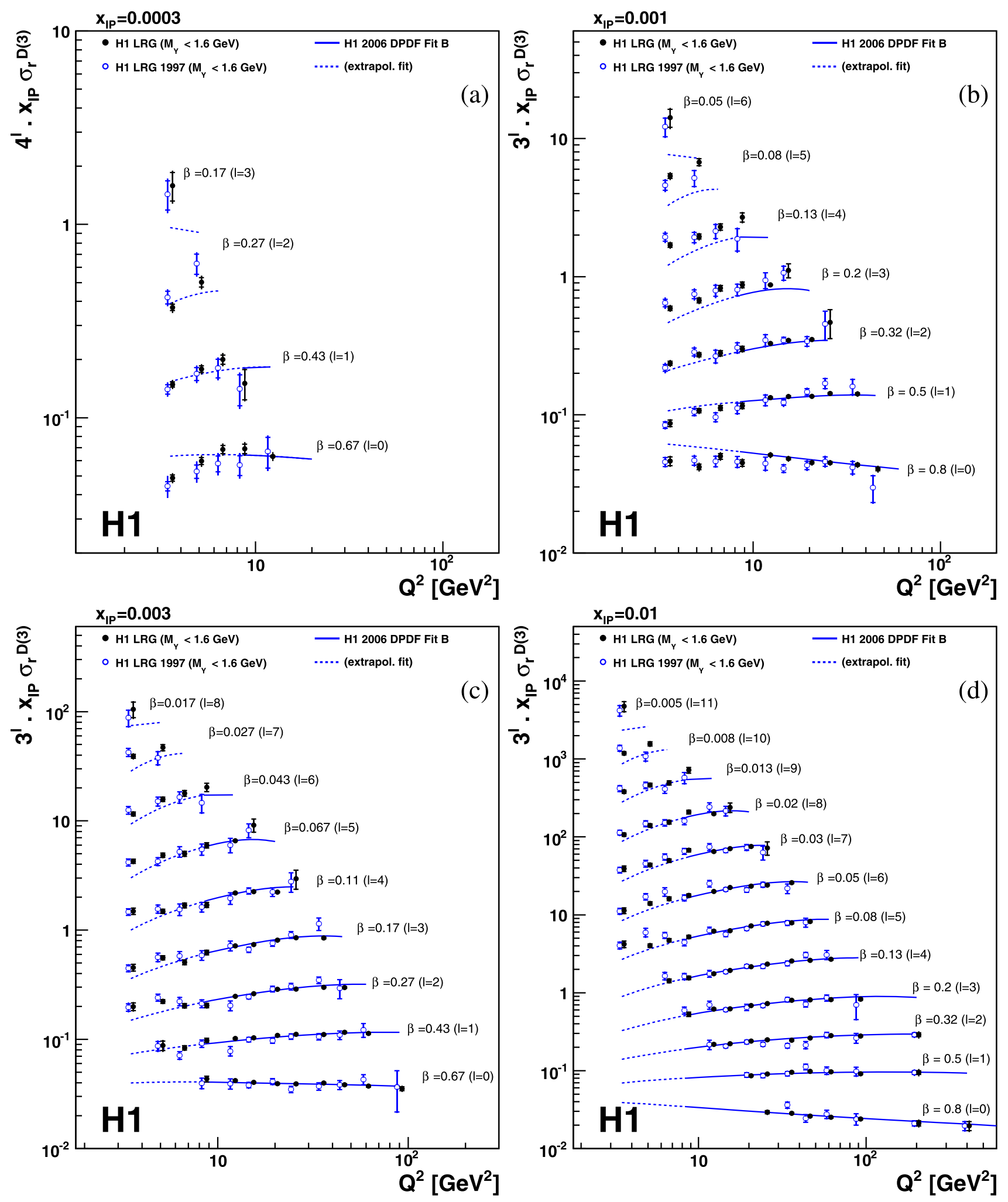

Fig. 7 The $Q^{2}$ dependence of the reduced diffractive cross section, multiplied by $x_{\mathbb{P}}$, at different fixed values of $x_{\mathbb{P}}=0.0003$ (a), 0.001 (b), 0.003 (c) and 0.01 (d), resulting from the combination of all data samples. The reduced cross section values are multiplied by a scaling factor, $4^{l}$ for $x_{\mathbb{P}}=0.0003$ and $3^{l}$ for $x_{\mathbb{P}}=0.003,0.001$ and 0.01 , with

$l$ values as indicated in parentheses. Previously published H1 measurements [10] are also displayed as open points. The measurements are displaced horizontally for better visibility. More details are explained in the caption of Fig. 3 
Fig. 8 The $\beta$ (a) and $Q^{2}$ (b) dependences of the reduced diffractive cross section, multiplied by $x_{\mathbb{P}}$, at a fixed value of $x_{\mathbb{P}}=0.03$, resulting from the combination of all data samples. Details are explained in the captions of Figs. 3 and 7

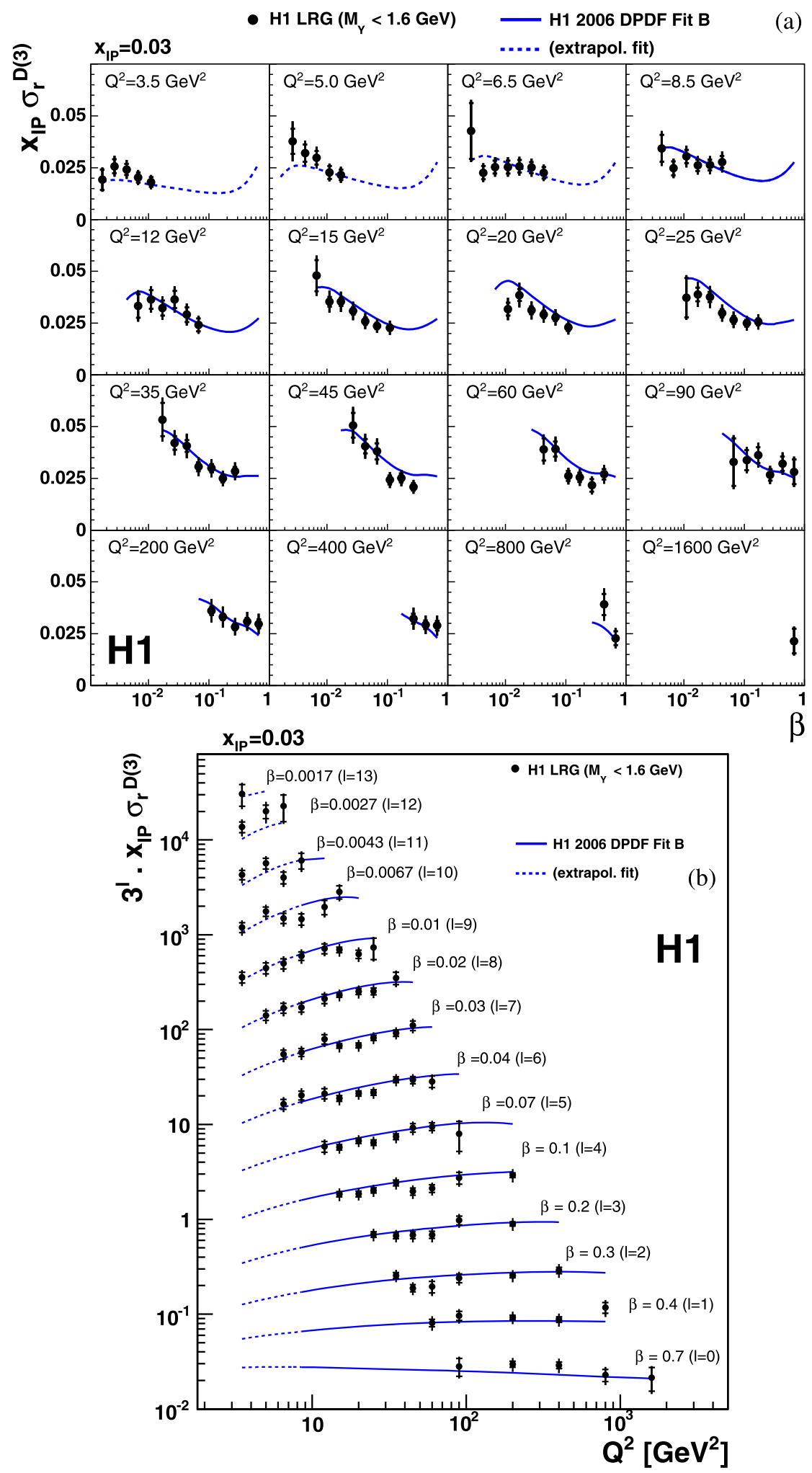

As the dipole model predictions correspond to the process $e p \rightarrow e X p$, they are rescaled by a factor of 1.20 according to Eq. (9). Both approaches give a good overall description of the measurements. In the low $Q^{2}$ range, for $Q^{2}<8.5 \mathrm{GeV}^{2}$, the dipole model, which includes saturation effects, seems to better describe the data, whereas for larger $\beta$ and for $x_{\mathbb{P}}=0.01$ it tends to underestimate the measured cross section. 
Table 2 The reduced diffractive cross section from combined H1 LRG data $x_{\mathbb{P}} \sigma_{r}{ }^{D(3)}$ quoted at fixed $Q^{2}, \beta$ and $x_{\mathbb{P}}$ (columns 1-4). The uncorrelated and statistical $\left(\delta_{\text {unc }}\right)$, correlated systematic $\left(\delta_{\text {sys }}\right)$, and total $\left(\delta_{\text {tot }}\right)$ uncertainties are given in columns 5 to 7 . All uncertainties are given in per cent. The overall normalisation uncertainty of $4 \%$ is not included. The table continues on the next pages

\begin{tabular}{|c|c|c|c|c|c|c|c|c|c|c|c|c|c|}
\hline & & & & & & & \\
\hline$x_{\mathbb{P}}$ & $\begin{array}{l}Q^{2} \\
{\left[\mathrm{GeV}^{2}\right]}\end{array}$ & $\beta$ & $x_{\mathbb{P}} \sigma_{r}^{D(3)}$ & $\begin{array}{l}\delta_{\text {unc }} \\
{[\%]}\end{array}$ & $\begin{array}{l}\delta_{\text {sys }} \\
{[\%]}\end{array}$ & $\begin{array}{l}\delta_{\text {tot }} \\
{[\%]}\end{array}$ & $\begin{array}{l}0.0010 \\
0.0010\end{array}$ & $\begin{array}{l}25.0 \\
25.0\end{array}$ & $\begin{array}{l}0.3200 \\
0.5000\end{array}$ & $\begin{array}{l}0.05186 \\
0.04764\end{array}$ & $\begin{array}{r}24.0 \\
2.0\end{array}$ & $\begin{array}{l}4.6 \\
3.4\end{array}$ & $\begin{array}{r}24.4 \\
4.0\end{array}$ \\
\hline & & & & & & & 0.0010 & 25.0 & 0.8000 & 0.04499 & 2.7 & 3.9 & 4.8 \\
\hline 0.0003 & 3.5 & 0.1700 & 0.02481 & 18.3 & 6.5 & 19.4 & 0.0010 & 35.0 & 0.5000 & 0.04718 & 2.6 & 3.6 & 4.4 \\
\hline 0.0003 & 3.5 & 0.2700 & 0.02327 & 4.4 & 4.7 & 6.4 & 0.0010 & 35.0 & 0.8000 & 0.04344 & 3.1 & 4.0 & 5.1 \\
\hline 0.0003 & 3.5 & 0.4300 & 0.03720 & 3.9 & 3.9 & 5.5 & 0.0010 & 45.0 & 0.8000 & 0.04048 & 3.7 & 4.5 & 5.8 \\
\hline 0.0003 & 3.5 & 0.6700 & 0.04880 & 4.2 & 4.5 & 6.1 & 0.0030 & 3.5 & 0.0170 & 0.01604 & 17.3 & 7.1 & 18.6 \\
\hline 0.0003 & 5.0 & 0.2700 & 0.03142 & 6.1 & 5.2 & 8.0 & 0.0030 & 3.5 & 0.0270 & 0.01785 & 5.0 & 4.6 & 6.8 \\
\hline 0.0003 & 5.0 & 0.4300 & 0.04465 & 4.6 & 4.2 & 6.2 & 0.0030 & 3.5 & 0.0430 & 0.01585 & 4.9 & 3.4 & 6.0 \\
\hline 0.0003 & 5.0 & 0.6700 & 0.05977 & 4.7 & 4.7 & 6.6 & 0.0030 & 3.5 & 0.0670 & 0.01758 & 4.8 & 3.6 & 6.0 \\
\hline 0.0003 & 6.5 & 0.4300 & 0.05005 & 6.0 & 5.1 & 7.8 & 0.0030 & 3.5 & 0.1100 & 0.01841 & 7.3 & 3.3 & 8.0 \\
\hline 0.0003 & $\begin{array}{l}6.5 \\
8.5\end{array}$ & $\begin{array}{l}0.6700 \\
0.4300\end{array}$ & $\begin{array}{l}0.06865 \\
0.03764\end{array}$ & $\begin{array}{r}5.4 \\
181\end{array}$ & $\begin{array}{l}4.8 \\
6.3\end{array}$ & $\begin{array}{r}7.2 \\
192\end{array}$ & 0.0030 & 3.5 & 0.1700 & 0.01678 & 7.7 & 3.3 & 8.4 \\
\hline $\begin{array}{l}0.0003 \\
0.0003\end{array}$ & $\begin{array}{l}8.5 \\
8.5\end{array}$ & $\begin{array}{l}0.4300 \\
0.6700\end{array}$ & $\begin{array}{l}0.03764 \\
0.06919\end{array}$ & $\begin{array}{r}18.1 \\
6.3\end{array}$ & $\begin{array}{l}6.3 \\
5.0\end{array}$ & $\begin{array}{r}19.2 \\
8.1\end{array}$ & 0.0030 & 3.5 & 0.2700 & 0.02215 & 9.0 & 4.6 & 10.1 \\
\hline 0.0003 & 12.0 & 0.6700 & 0.06314 & 1.9 & 5.0 & 5.3 & 0.0030 & 5.0 & 0.0270 & 0.02143 & 7.0 & 4.7 & 8.4 \\
\hline 0.0010 & 3.5 & 0.0500 & 0.01945 & 15.7 & 7.3 & 17.3 & 0.0030 & 5.0 & 0.0430 & 0.02163 & 5.3 & 3.9 & 6.5 \\
\hline 0.0010 & 3.5 & 0.0800 & 0.02203 & 4.4 & 5.1 & 6.7 & 0.0030 & 5.0 & 0.0670 & 0.01994 & 5.1 & 3.5 & 6.2 \\
\hline 0.0010 & 3.5 & 0.1300 & 0.02087 & 4.2 & 4.2 & 5.9 & 0.0030 & 5.0 & 0.1100 & 0.01834 & 5.1 & 3.4 & 6.1 \\
\hline 0.0010 & 3.5 & 0.2000 & 0.02188 & 4.3 & 4.1 & 6.0 & 0.0030 & 5.0 & 0.1700 & 0.02068 & 4.9 & 3.5 & 6.0 \\
\hline 0.0010 & 3.5 & 0.3200 & 0.02622 & 4.1 & 3.9 & 5.7 & 0.0030 & 5.0 & 0.2700 & 0.02472 & 4.6 & 3.5 & 5.8 \\
\hline 0.0010 & 3.5 & 0.5000 & 0.02897 & 6.2 & 3.3 & 7.0 & 0.0030 & 5.0 & 0.4300 & 0.02922 & 11.2 & 5.6 & 12.5 \\
\hline 0.0010 & 3.5 & 0.8000 & 0.04622 & 7.9 & 4.6 & 9.1 & 0.0030 & 6.5 & 0.0430 & 0.02452 & 6.4 & 3.8 & 7.5 \\
\hline 0.0010 & 5.0 & 0.0800 & 0.02777 & 6.1 & 4.3 & 7.4 & 0.0030 & 6.5 & 0.0670 & 0.02060 & 5.9 & 3.4 & 6.8 \\
\hline 0.0010 & 5.0 & 0.1300 & 0.02411 & 4.7 & 4.3 & 6.4 & 0.0030 & 6.5 & 0.1100 & 0.02079 & 5.7 & 3.4 & 6.7 \\
\hline 0.0010 & 5.0 & 0.2000 & 0.02495 & 4.5 & 4.1 & 6.1 & 0.0030 & 6.5 & 0.1700 & 0.01880 & 5.4 & 3.3 & 6.3 \\
\hline 0.0010 & 5.0 & 0.3200 & 0.03026 & 4.3 & 4.0 & 5.9 & 0.0030 & 6.5 & 0.2700 & 0.02256 & 5.2 & 3.4 & 6.2 \\
\hline 0.0010 & 5.0 & 0.5000 & 0.03570 & 4.3 & 3.3 & 5.4 & 0.0030 & 6.5 & 0.4300 & 0.02785 & 5.2 & 3.5 & 6.3 \\
\hline 0.0010 & $\begin{array}{l}5.0 \\
6.5\end{array}$ & $\begin{array}{l}0.8000 \\
0.1300\end{array}$ & $\begin{array}{l}0.04197 \\
0.02825\end{array}$ & $\begin{array}{l}5.4 \\
5.8\end{array}$ & $\begin{array}{l}5.4 \\
3.9\end{array}$ & $\begin{array}{l}7.6 \\
7.0\end{array}$ & 0.0030 & 8.5 & 0.0430 & 0.02783 & 9.2 & 4.2 & 10.1 \\
\hline $\begin{array}{l}0.0010 \\
0.0010\end{array}$ & $\begin{array}{l}6.5 \\
6.5\end{array}$ & $\begin{array}{l}0.1300 \\
0.2000\end{array}$ & $\begin{array}{l}0.02825 \\
0.03057\end{array}$ & $\begin{array}{l}5.8 \\
5.0\end{array}$ & $\begin{array}{l}3.9 \\
4.1\end{array}$ & $\begin{array}{l}7.0 \\
6.5\end{array}$ & 0.0030 & 8.5 & 0.0670 & 0.02460 & 6.1 & 3.4 & 6.9 \\
\hline $\begin{array}{l}0.0010 \\
0.0010\end{array}$ & 6.5 & 0.3200 & 0.03104 & 5.1 & $\begin{array}{l}4.1 \\
3.7\end{array}$ & 6.2 & 0.0030 & 8.5 & 0.1100 & 0.02097 & 5.9 & 3.5 & 6.8 \\
\hline 0.0010 & 6.5 & 0.5000 & 0.03740 & 4.7 & 3.5 & 5.9 & 0.0030 & 8.5 & 0.1700 & 0.02308 & 5.3 & 3.3 & 6.3 \\
\hline 0.0010 & 6.5 & 0.8000 & 0.05006 & 5.3 & 5.2 & 7.4 & 0.0030 & 8.5 & 0.2700 & 0.02265 & 5.1 & 3.3 & 6.1 \\
\hline 0.0010 & 8.5 & 0.1300 & 0.03321 & 8.0 & 4.9 & 9.4 & 0.0030 & 8.5 & 0.4300 & 0.03263 & 4.9 & 3.3 & 5.9 \\
\hline 0.0010 & 8.5 & 0.2000 & 0.03233 & 5.2 & 3.8 & 6.4 & 0.0030 & 8.5 & 0.6700 & 0.04341 & 6.5 & 3.7 & 7.4 \\
\hline 0.0010 & 8.5 & 0.3200 & 0.03332 & 4.9 & 3.6 & 6.1 & 0.0030 & 12.0 & 0.0670 & 0.02712 & 2.3 & 3.4 & 4.1 \\
\hline 0.0010 & 8.5 & 0.5000 & 0.03871 & 5.3 & 3.7 & 6.4 & 0.0030 & 12.0 & 0.1100 & 0.02698 & 2.2 & 3.3 & 3.9 \\
\hline 0.0010 & 8.5 & 0.8000 & 0.04488 & 6.1 & 4.6 & 7.6 & 0.0030 & 12.0 & 0.1700 & 0.02655 & 2.2 & 3.1 & 3.8 \\
\hline 0.0010 & 12.0 & 0.2000 & 0.03227 & 1.8 & 3.3 & 3.8 & 0.0030 & 12.0 & 0.2700 & 0.02751 & 2.3 & 3.2 & 4.0 \\
\hline 0.0010 & 12.0 & 0.3200 & 0.03650 & 1.9 & 3.2 & 3.7 & 0.0030 & 12.0 & 0.4300 & 0.03388 & 2.3 & 3.2 & 4.0 \\
\hline 0.0010 & 12.0 & 0.5000 & 0.04438 & 2.3 & 3.2 & 3.9 & 0.0030 & 12.0 & 0.6700 & 0.04193 & 2.6 & 3.3 & 4.2 \\
\hline 0.0010 & 12.0 & 0.8000 & 0.05118 & 2.7 & 4.4 & 5.1 & 0.0030 & 15.0 & 0.0670 & 0.03764 & 14.2 & 4.7 & 14.9 \\
\hline 0.0010 & 15.0 & 0.2000 & 0.04107 & 11.8 & 4.5 & 12.6 & 0.0030 & 15.0 & 0.1100 & 0.02780 & 2.1 & 3.4 & 3.9 \\
\hline 0.0010 & 15.0 & 0.3200 & 0.03840 & 1.8 & 3.2 & 3.6 & 0.0030 & 15.0 & 0.1700 & 0.02732 & 2.1 & 3.2 & 3.8 \\
\hline 0.0010 & $\begin{array}{l}15.0 \\
15.0\end{array}$ & $\begin{array}{l}0.5000 \\
0.8000\end{array}$ & $\begin{array}{l}0.04522 \\
0.04816\end{array}$ & $\begin{array}{l}2.1 \\
2.7\end{array}$ & 3.3 & $\begin{array}{l}3.9 \\
5.0\end{array}$ & 0.0030 & 15.0 & 0.2700 & 0.02903 & 2.0 & 3.1 & 3.7 \\
\hline $\begin{array}{l}0.0010 \\
0.0010\end{array}$ & $\begin{array}{l}15.0 \\
20.0\end{array}$ & $\begin{array}{l}0.8000 \\
0.3200\end{array}$ & $\begin{array}{l}0.04816 \\
0.03892\end{array}$ & $\begin{array}{l}2.7 \\
1.9\end{array}$ & $\begin{array}{l}4.2 \\
3.2\end{array}$ & $\begin{array}{l}5.0 \\
3.8\end{array}$ & 0.0030 & 15.0 & 0.4300 & 0.03449 & 2.2 & 3.1 & 3.8 \\
\hline 0.0010 & 20.0 & 0.5000 & 0.04528 & 2.1 & 3.2 & 3.9 & 0.0030 & 15.0 & 0.6700 & 0.04031 & 2.5 & 3.2 & 4.1 \\
\hline 0.0010 & 20.0 & 0.8000 & 0.04510 & 2.7 & 4.1 & 4.9 & 0.0030 & 20.0 & 0.1100 & 0.02754 & 2.3 & 3.3 & 4.1 \\
\hline
\end{tabular}

Table 3 The reduced diffractive cross section from combined H1 LRG data $x_{\mathbb{P}} \sigma_{r}{ }^{D(3)}$ quoted at fixed $Q^{2}, \beta$ and $x_{\mathbb{P}}$, continued from Table 2

\begin{tabular}{lllllll}
\hline$x_{\mathbb{P}}$ & $Q^{2}$ & $\beta$ & $x_{\mathbb{P}} \sigma_{r}^{D(3)}$ & $\delta_{\text {unc }}$ & $\delta_{\text {sys }}$ & $\delta_{\text {to }}$ \\
& {$\left[\mathrm{GeV}^{2}\right]$} & & & {$[\%]$} & {$[\%]$} & {$[\%$}
\end{tabular}

$\delta_{\text {tot }}$

4

.0 1 8 
Table 4 The reduced diffractive cross section from combined H1 LRG data $x_{\mathbb{P}} \sigma_{r}{ }^{D(3)}$ quoted at fixed $Q^{2}, \beta$ and $x_{\mathbb{P}}$, continued from Table 2

\begin{tabular}{|c|c|c|c|c|c|c|c|c|c|c|c|c|c|}
\hline$x_{\mathbb{P}}$ & $\begin{array}{l}Q^{2} \\
{\left[\mathrm{GeV}^{2}\right]}\end{array}$ & $\beta$ & $x_{\mathbb{P}} \sigma_{r}^{D(3)}$ & $\begin{array}{l}\delta_{\text {unc }} \\
{[\%]}\end{array}$ & $\begin{array}{l}\delta_{\text {sys }} \\
{[\%]}\end{array}$ & $\begin{array}{l}\delta_{\text {tot }} \\
{[\%]}\end{array}$ & $x_{\mathbb{P}}$ & $\begin{array}{l}Q^{2} \\
{\left[\mathrm{GeV}^{2}\right]}\end{array}$ & $\beta$ & $x_{\mathbb{P}} \sigma_{r}^{D(3)}$ & $\begin{array}{l}\delta_{\text {unc }} \\
{[\%]}\end{array}$ & $\begin{array}{l}\delta_{\text {sys }} \\
{[\%]}\end{array}$ & $\begin{array}{l}\delta_{\text {tot }} \\
{[\%]}\end{array}$ \\
\hline 0.0030 & 20.0 & 0.1700 & 0.02996 & 2.0 & 3.2 & 3.8 & 0.0100 & 12.0 & 0.0320 & 0.02966 & 3.8 & 3.2 & 5.0 \\
\hline 0.0030 & 20.0 & 0.2700 & 0.03194 & 1.9 & 3.0 & 3.6 & 0.0100 & 12.0 & 0.0500 & 0.02732 & 3.7 & 3.2 & 4.9 \\
\hline 0.0030 & 20.0 & 0.4300 & 0.03618 & 2.0 & 3.1 & 3.7 & 0.0100 & 12.0 & 0.0800 & 0.02545 & 3.6 & 3.2 & 4.8 \\
\hline 0.0030 & 20.0 & 0.6700 & 0.03927 & 2.4 & 3.3 & 4.1 & 0.0100 & 12.0 & 0.1300 & 0.02165 & 3.8 & 3.2 & 4.9 \\
\hline 0.0030 & 25.0 & 0.1100 & 0.03645 & 20.2 & 5.4 & 20.9 & 0.0100 & 12.0 & 0.2000 & 0.02283 & 4.2 & 3.1 & 5.2 \\
\hline 0.0030 & 25.0 & 0.1700 & 0.03156 & 2.1 & 3.0 & 3.7 & 0.0100 & 12.0 & 0.3200 & 0.02420 & 5.1 & 3.1 & 6.0 \\
\hline 0.0030 & 25.0 & 0.2700 & 0.03205 & 1.9 & 3.0 & 3.6 & 0.0100 & 15.0 & 0.0200 & 0.03639 & 14.5 & 5.8 & 15.6 \\
\hline 0.0030 & 25.0 & 0.4300 & 0.03706 & 2.0 & 3.0 & 3.6 & 0.0100 & 15.0 & 0.0320 & 0.03226 & 3.2 & 3.4 & 4.7 \\
\hline 0.0030 & 25.0 & 0.6700 & 0.03909 & 2.4 & 3.4 & 4.2 & 0.0100 & 15.0 & 0.0500 & 0.03067 & 3.1 & 3.3 & 4.6 \\
\hline 0.0030 & 35.0 & 0.1700 & 0.03132 & 2.5 & 3.3 & 4.1 & 0.0100 & 15.0 & 0.0800 & 0.02573 & 3.2 & 3.3 & 4.6 \\
\hline 0.0030 & 35.0 & 0.2700 & 0.03330 & 2.0 & 2.9 & 3.6 & 0.0100 & 15.0 & 0.1300 & 0.02381 & 3.0 & 3.3 & 4.5 \\
\hline 0.0030 & 35.0 & 0.4300 & 0.03691 & 2.1 & 3.1 & 3.7 & 0.0100 & 15.0 & 0.2000 & 0.02299 & 3.0 & 3.3 & 4.5 \\
\hline 0.0030 & 35.0 & 0.6700 & 0.03975 & 2.5 & 3.7 & 4.4 & 0.0100 & 15.0 & 0.3200 & 0.02456 & 3.3 & 3.1 & 4.5 \\
\hline 0.0030 & 45.0 & 0.2700 & 0.03306 & 2.4 & 3.0 & 3.9 & 0.0100 & 20.0 & 0.0320 & 0.03445 & 4.0 & 3.3 & 5.2 \\
\hline 0.0030 & 45.0 & 0.4300 & 0.03872 & 2.3 & 3.0 & 3.8 & 0.0100 & 20.0 & 0.0500 & 0.03209 & 3.3 & 3.4 & 4.8 \\
\hline 0.0030 & 45.0 & 0.6700 & 0.03844 & 2.8 & 3.7 & 4.6 & 0.0100 & 20.0 & 0.0800 & 0.02971 & 3.5 & 3.3 & 4.8 \\
\hline 0.0030 & 60.0 & 0.4300 & 0.03776 & 2.7 & 3.1 & 4.1 & 0.0100 & 20.0 & 0.1300 & 0.02658 & 3.1 & 3.2 & 4.5 \\
\hline 0.0030 & 60.0 & 0.6700 & 0.03728 & 3.1 & 3.7 & 4.8 & 0.0100 & 20.0 & 0.2000 & 0.02542 & 3.4 & 3.2 & 4.7 \\
\hline 0.0030 & 90.0 & 0.6700 & 0.03532 & 5.4 & 4.4 & 6.9 & 0.0100 & 20.0 & 0.3200 & 0.02663 & 3.1 & 3.2 & 4.4 \\
\hline 0.0100 & 3.5 & 0.0050 & 0.02678 & 16.0 & 6.1 & 17.1 & 0.0100 & 20.0 & 0.5000 & 0.02870 & 3.7 & 3.2 & 4.8 \\
\hline 0.0100 & 3.5 & 0.0080 & 0.02007 & 6.7 & 4.3 & 7.9 & 0.0100 & 25.0 & 0.0320 & 0.03306 & 19.8 & 6.4 & 20.8 \\
\hline 0.0100 & 3.5 & 0.0130 & 0.01938 & 6.8 & 3.9 & 7.9 & 0.0100 & 25.0 & 0.0500 & 0.03307 & 3.2 & 3.5 & 4.8 \\
\hline 0.0100 & 3.5 & 0.0200 & 0.01632 & 6.3 & 3.5 & 7.2 & 0.0100 & 25.0 & 0.0800 & 0.03202 & 3.2 & 3.4 & 4.7 \\
\hline 0.0100 & 3.5 & 0.0320 & 0.01795 & 9.3 & 4.0 & 10.1 & 0.0100 & 25.0 & 0.1300 & 0.02889 & 3.2 & 3.4 & 4.6 \\
\hline 0.0100 & 3.5 & 0.0500 & 0.01554 & 9.8 & 3.7 & 10.5 & 0.0100 & 25.0 & 0.2000 & 0.02686 & 3.0 & 3.3 & 4.5 \\
\hline 0.0100 & 3.5 & 0.0800 & 0.01729 & 11.0 & 4.4 & 11.8 & 0.0100 & 25.0 & 0.3200 & 0.02769 & 3.1 & 3.4 & 4.6 \\
\hline 0.0100 & 5.0 & 0.0080 & 0.02647 & 7.5 & 4.8 & 8.9 & 0.0100 & 25.0 & 0.5000 & 0.03028 & 3.4 & 3.3 & 4.7 \\
\hline 0.0100 & 5.0 & 0.0130 & 0.02361 & 6.7 & 4.0 & 7.8 & 0.0100 & 25.0 & 0.8000 & 0.02928 & 7.0 & 3.8 & 7.9 \\
\hline 0.0100 & 5.0 & 0.0200 & 0.02137 & 6.4 & 3.6 & 7.4 & 0.0100 & 35.0 & 0.0500 & 0.03551 & 4.1 & 3.5 & 5.3 \\
\hline 0.0100 & 5.0 & 0.0320 & 0.02000 & 6.3 & 3.5 & 7.2 & 0.0100 & 35.0 & 0.0800 & 0.03243 & 3.8 & 3.3 & 5.0 \\
\hline 0.0100 & 5.0 & 0.0500 & 0.01922 & 6.3 & 3.5 & 7.2 & 0.0100 & 35.0 & 0.1300 & 0.03161 & 3.2 & 3.3 & 4.6 \\
\hline 0.0100 & 5.0 & 0.0800 & 0.01657 & 6.9 & 3.9 & 8.0 & 0.0100 & 35.0 & 0.2000 & 0.02963 & 3.3 & 3.1 & 4.5 \\
\hline 0.0100 & 6.5 & 0.0130 & 0.02516 & 7.2 & 3.8 & 8.1 & 0.0100 & 35.0 & 0.3200 & 0.02729 & 3.2 & 3.7 & 4.9 \\
\hline 0.0100 & 6.5 & 0.0200 & 0.02356 & 6.9 & 3.3 & 7.7 & 0.0100 & 35.0 & 0.5000 & 0.03171 & 3.5 & 3.1 & 4.7 \\
\hline 0.0100 & 6.5 & 0.0320 & 0.02270 & 6.4 & 3.3 & 7.2 & 0.0100 & 35.0 & 0.8000 & 0.02840 & 4.3 & 3.5 & 5.5 \\
\hline 0.0100 & 6.5 & 0.0500 & 0.02205 & 6.8 & 3.5 & 7.6 & 0.0100 & 45.0 & 0.0800 & 0.03368 & 4.1 & 3.3 & 5.3 \\
\hline 0.0100 & 6.5 & 0.0800 & 0.01938 & 5.9 & 3.6 & 6.9 & 0.0100 & 45.0 & 0.1300 & 0.03212 & 3.4 & 3.2 & 4.6 \\
\hline 0.0100 & 6.5 & 0.1300 & 0.01757 & 6.7 & 3.4 & 7.5 & 0.0100 & 45.0 & 0.2000 & 0.02994 & 3.4 & 3.2 & 4.7 \\
\hline 0.0100 & 8.5 & 0.0130 & 0.03654 & 9.2 & 4.0 & 10.0 & 0.0100 & 45.0 & 0.3200 & 0.02910 & 3.3 & 3.5 & 4.8 \\
\hline 0.0100 & 8.5 & 0.0200 & 0.03174 & 6.2 & 3.8 & 7.3 & 0.0100 & 45.0 & 0.5000 & 0.03255 & 3.7 & 3.0 & 4.8 \\
\hline 0.0100 & 8.5 & 0.0320 & 0.03085 & 5.8 & 3.4 & 6.7 & 0.0100 & 45.0 & 0.8000 & 0.02606 & 4.5 & 3.5 & 5.7 \\
\hline 0.0100 & 8.5 & 0.0500 & 0.02431 & 6.1 & 3.2 & 6.9 & 0.0100 & 60.0 & 0.1300 & 0.03316 & 4.1 & 3.1 & 5.2 \\
\hline 0.0100 & 8.5 & 0.0800 & 0.02142 & 5.9 & 3.5 & 6.8 & 0.0100 & 60.0 & 0.2000 & 0.03013 & 3.3 & 3.3 & 4.7 \\
\hline 0.0100 & 8.5 & 0.1300 & 0.01919 & 6.1 & 3.6 & 7.1 & 0.0100 & 60.0 & 0.3200 & 0.03138 & 3.4 & 3.1 & 4.6 \\
\hline 0.0100 & 8.5 & 0.2000 & 0.01961 & 7.2 & 3.3 & 7.9 & 0.0100 & 60.0 & 0.5000 & 0.03225 & 3.6 & 3.7 & 5.2 \\
\hline 0.0100 & 12.0 & 0.0200 & 0.03014 & 3.9 & 3.3 & 5.1 & 0.0100 & 60.0 & 0.8000 & 0.02516 & 4.0 & 3.7 & 5.4 \\
\hline
\end{tabular}

Table 5 The reduced diffractive cross section from combined H1 LRG data $x_{\mathbb{P}} \sigma_{r}{ }^{D(3)}$ quoted at fixed $Q^{2}, \beta$ and $x_{\mathbb{P}}$, continued from Table 2

\section{9} 8 (n) 
Table 6 The reduced diffractive cross section from combined H1 LRG data $x_{\mathbb{P}} \sigma_{r}{ }^{D(3)}$ quoted at fixed $Q^{2}, \beta$ and $x_{\mathbb{P}}$, continued from Table 2

\begin{tabular}{|c|c|c|c|c|c|c|}
\hline$x_{\mathbb{P}}$ & $\begin{array}{l}Q^{2} \\
{\left[\mathrm{GeV}^{2}\right]}\end{array}$ & $\beta$ & $x_{\mathbb{P}} \sigma_{r}^{D(3)}$ & $\begin{array}{l}\delta_{\text {unc }} \\
{[\%]}\end{array}$ & $\begin{array}{l}\delta_{\text {sys }} \\
{[\%]}\end{array}$ & $\begin{array}{l}\delta_{\text {tot }} \\
{[\%]}\end{array}$ \\
\hline 0.0100 & 90.0 & 0.2000 & 0.03061 & 5.0 & 3.5 & 6.2 \\
\hline 0.0100 & 90.0 & 0.3200 & 0.03095 & 4.3 & 3.1 & 5.2 \\
\hline 0.0100 & 90.0 & 0.5000 & 0.03039 & 3.8 & 3.3 & 5.1 \\
\hline 0.0100 & 90.0 & 0.8000 & 0.02396 & 4.3 & 3.6 & 5.7 \\
\hline 0.0100 & 200.0 & 0.3200 & 0.03210 & 6.8 & 8.5 & 10.9 \\
\hline 0.0100 & 200.0 & 0.5000 & 0.03150 & 6.4 & 8.8 & 10.9 \\
\hline 0.0100 & 200.0 & 0.8000 & 0.02110 & 8.7 & 8.0 & 11.8 \\
\hline 0.0100 & 400.0 & 0.8000 & 0.01960 & 13.8 & 9.6 & 16.7 \\
\hline 0.0300 & 3.5 & 0.0017 & 0.01919 & 29.3 & 8.7 & 30.6 \\
\hline 0.0300 & 3.5 & 0.0027 & 0.02575 & 18.0 & 8.6 & 19.9 \\
\hline 0.0300 & 3.5 & 0.0043 & 0.02418 & 17.0 & 7.8 & 18.7 \\
\hline 0.0300 & 3.5 & 0.0067 & 0.02030 & 16.9 & 6.9 & 18.2 \\
\hline 0.0300 & 3.5 & 0.0110 & 0.01811 & 17.6 & 6.8 & 18.9 \\
\hline 0.0300 & 5.0 & 0.0027 & 0.03776 & 21.1 & 14.3 & 25.5 \\
\hline 0.0300 & 5.0 & 0.0043 & 0.03206 & 17.8 & 6.3 & 18.9 \\
\hline 0.0300 & 5.0 & 0.0067 & 0.02984 & 16.2 & 7.1 & 17.7 \\
\hline 0.0300 & 5.0 & 0.0110 & 0.02269 & 17.7 & 6.4 & 18.9 \\
\hline 0.0300 & 5.0 & 0.0170 & 0.02157 & 16.7 & 7.3 & 18.2 \\
\hline 0.0300 & 6.5 & 0.0027 & 0.04277 & 34.1 & 8.7 & 35.2 \\
\hline 0.0300 & 6.5 & 0.0043 & 0.02261 & 18.4 & 7.9 & 20.1 \\
\hline 0.0300 & 6.5 & 0.0067 & 0.02536 & 17.3 & 7.0 & 18.6 \\
\hline 0.0300 & 6.5 & 0.0110 & 0.02534 & 17.4 & 7.0 & 18.7 \\
\hline 0.0300 & 6.5 & 0.0170 & 0.02571 & 17.0 & 5.5 & 17.9 \\
\hline 0.0300 & 6.5 & 0.0270 & 0.02512 & 16.3 & 6.6 & 17.6 \\
\hline 0.0300 & 6.5 & 0.0430 & 0.02256 & 16.8 & 6.1 & 17.9 \\
\hline 0.0300 & 8.5 & 0.0043 & 0.03435 & 23.1 & 8.8 & 24.7 \\
\hline 0.0300 & 8.5 & 0.0067 & 0.02474 & 18.6 & 5.1 & 19.3 \\
\hline 0.0300 & 8.5 & 0.0110 & 0.03042 & 16.1 & 5.7 & 17.1 \\
\hline 0.0300 & 8.5 & 0.0170 & 0.02617 & 15.8 & 6.3 & 17.0 \\
\hline 0.0300 & 8.5 & 0.0270 & 0.02631 & 15.3 & 6.4 & 16.6 \\
\hline 0.0300 & 8.5 & 0.0430 & 0.02782 & 17.1 & 6.1 & 18.1 \\
\hline 0.0300 & 12.0 & 0.0067 & 0.03331 & 22.0 & 5.8 & 22.7 \\
\hline 0.0300 & 12.0 & 0.0110 & 0.03641 & 16.7 & 4.9 & 17.4 \\
\hline 0.0300 & 12.0 & 0.0170 & 0.03224 & 16.3 & 6.7 & 17.6 \\
\hline 0.0300 & 12.0 & 0.0270 & 0.03637 & 16.1 & 6.5 & 17.4 \\
\hline 0.0300 & 12.0 & 0.0430 & 0.02906 & 17.5 & 5.5 & 18.4 \\
\hline 0.0300 & 12.0 & 0.0670 & 0.02413 & 17.6 & 5.2 & 18.3 \\
\hline 0.0300 & 15.0 & 0.0067 & 0.04792 & 19.4 & 6.4 & 20.4 \\
\hline 0.0300 & 15.0 & 0.0110 & 0.03531 & 13.7 & 6.6 & 15.2 \\
\hline 0.0300 & 15.0 & 0.0170 & 0.03527 & 12.6 & 6.4 & 14.1 \\
\hline 0.0300 & 15.0 & 0.0270 & 0.03085 & 13.3 & 5.9 & 14.5 \\
\hline 0.0300 & 15.0 & 0.0430 & 0.02592 & 13.4 & 7.1 & 15.2 \\
\hline 0.0300 & 15.0 & 0.0670 & 0.02366 & 13.3 & 5.9 & 14.5 \\
\hline 0.0300 & 15.0 & 0.1100 & 0.02278 & 13.7 & 6.4 & 15.2 \\
\hline 0.0300 & 20.0 & 0.0110 & 0.03178 & 15.6 & 7.2 & 17.2 \\
\hline 0.0300 & 20.0 & 0.0170 & 0.03851 & 14.0 & 6.2 & 15.4 \\
\hline
\end{tabular}

Table 7 The reduced diffractive cross section from combined H1 LRG data $x_{\mathbb{P}} \sigma_{r}{ }^{D(3)}$ quoted at fixed $Q^{2}, \beta$ and $x_{\mathbb{P}}$, continued from Table 2

\begin{tabular}{lllllll}
\hline$x_{\mathbb{P}}$ & $Q^{2}$ & $\beta$ & $x_{\mathbb{P}} \sigma_{r}^{D(3)}$ & $\delta_{\text {unc }}$ & $\delta_{\text {sys }}$ & $\delta_{\text {to }}$
\end{tabular}

\begin{tabular}{rrrrrrr} 
& {$\left[\mathrm{GeV}^{2}\right]$} & & & {$[\%]$} & {$[\%]$} & {$[\%]$} \\
\hline 0.0300 & 20.0 & 0.0270 & 0.03118 & 12.9 & 5.5 & 14.1
\end{tabular}

$\begin{array}{lllllll}0.0300 & 20.0 & 0.0430 & 0.02917 & 12.9 & 5.5 & 14.0\end{array}$

$\begin{array}{lllllll}0.0300 & 20.0 & 0.0670 & 0.02773 & 13.0 & 5.5 & 14.1\end{array}$

$\begin{array}{lllllll}0.0300 & 20.0 & 0.1100 & 0.02288 & 13.5 & 5.8 & 14.7\end{array}$

$\begin{array}{lllllll}0.0300 & 25.0 & 0.0110 & 0.03729 & 28.0 & 7.4 & 29.0\end{array}$

$\begin{array}{lllllll}0.0300 & 25.0 & 0.0170 & 0.03875 & 14.3 & 6.3 & 15.6\end{array}$

$\begin{array}{lllllll}0.0300 & 25.0 & 0.0270 & 0.03755 & 13.1 & 5.6 & 14.3\end{array}$

$\begin{array}{lllllll}0.0300 & 25.0 & 0.0430 & 0.02978 & 13.1 & 4.7 & 14.0\end{array}$

$\begin{array}{lllllll}0.0300 & 25.0 & 0.0670 & 0.02655 & 13.8 & 6.6 & 15.3\end{array}$

$\begin{array}{lllllll}0.0300 & 25.0 & 0.1100 & 0.02491 & 13.1 & 6.4 & 14.5\end{array}$

$\begin{array}{lllllll}0.0300 & 25.0 & 0.1700 & 0.02562 & 13.3 & 6.4 & 14.7\end{array}$

$\begin{array}{lllllll}0.0300 & 35.0 & 0.0170 & 0.05337 & 18.9 & 6.1 & 19.9\end{array}$

$\begin{array}{lllllll}0.0300 & 35.0 & 0.0270 & 0.04213 & 13.8 & 5.0 & 14.6\end{array}$

$\begin{array}{lllllll}0.0300 & 35.0 & 0.0430 & 0.04063 & 14.0 & 4.6 & 14.7\end{array}$

$\begin{array}{lllllll}0.0300 & 35.0 & 0.0670 & 0.03063 & 13.6 & 6.0 & 14.8\end{array}$

$\begin{array}{lllllll}0.0300 & 35.0 & 0.1100 & 0.02992 & 13.4 & 6.3 & 14.8\end{array}$

$\begin{array}{lllllll}0.0300 & 35.0 & 0.1700 & 0.02493 & 13.8 & 6.2 & 15.1\end{array}$

$\begin{array}{lllllll}0.0300 & 35.0 & 0.2700 & 0.02840 & 13.5 & 6.6 & 15.1\end{array}$

$\begin{array}{lllllll}0.0300 & 45.0 & 0.0270 & 0.05064 & 17.0 & 4.9 & 17.6\end{array}$

$\begin{array}{lllllll}0.0300 & 45.0 & 0.0430 & 0.04048 & 14.3 & 4.4 & 15.0\end{array}$

$\begin{array}{lllllll}0.0300 & 45.0 & 0.0670 & 0.03804 & 15.4 & 6.1 & 16.5\end{array}$

$\begin{array}{lllllll}0.0300 & 45.0 & 0.1100 & 0.02427 & 14.6 & 6.6 & 16.0\end{array}$

$\begin{array}{lllllll}0.0300 & 45.0 & 0.1700 & 0.02521 & 14.2 & 7.1 & 15.9\end{array}$

$\begin{array}{lllllll}0.0300 & 45.0 & 0.2700 & 0.02092 & 14.4 & 6.5 & 15.8\end{array}$

$\begin{array}{lllllll}0.0300 & 60.0 & 0.0430 & 0.03900 & 17.7 & 5.7 & 18.6\end{array}$

$\begin{array}{lllllll}0.0300 & 60.0 & 0.0670 & 0.03913 & 14.7 & 5.2 & 15.6\end{array}$

$\begin{array}{lllllll}0.0300 & 60.0 & 0.1100 & 0.02613 & 14.6 & 5.5 & 15.6\end{array}$

$\begin{array}{lllllll}0.0300 & 60.0 & 0.1700 & 0.02548 & 14.3 & 8.2 & 16.5\end{array}$

$\begin{array}{lllllll}0.0300 & 60.0 & 0.2700 & 0.02165 & 18.2 & 8.5 & 20.1\end{array}$

$\begin{array}{lllllll}0.0300 & 60.0 & 0.4300 & 0.02698 & 14.7 & 8.7 & 17.1\end{array}$

$\begin{array}{lllllll}0.0300 & 90.0 & 0.0670 & 0.03286 & 39.0 & 6.9 & 39.6\end{array}$

$\begin{array}{lllllll}0.0300 & 90.0 & 0.1100 & 0.03379 & 18.5 & 4.5 & 19.1\end{array}$

$\begin{array}{lllllll}0.0300 & 90.0 & 0.1700 & 0.03622 & 15.6 & 6.0 & 16.7\end{array}$

$\begin{array}{lllllll}0.0300 & 90.0 & 0.2700 & 0.02668 & 15.4 & 5.5 & 16.4\end{array}$

$\begin{array}{lllllll}0.0300 & 90.0 & 0.4300 & 0.03214 & 16.1 & 5.7 & 17.1\end{array}$

$\begin{array}{lllllll}0.0300 & 90.0 & 0.6700 & 0.02818 & 24.5 & 7.9 & 25.8\end{array}$

$\begin{array}{lllllll}0.0300 & 200.0 & 0.1100 & 0.03610 & 12.5 & 9.8 & 15.9\end{array}$

$\begin{array}{lllllll}0.0300 & 200.0 & 0.1700 & 0.03310 & 12.1 & 9.5 & 15.4\end{array}$

$\begin{array}{lllllll}0.0300 & 200.0 & 0.2700 & 0.02830 & 12.3 & 8.4 & 14.9\end{array}$

$\begin{array}{lllllll}0.0300 & 200.0 & 0.4300 & 0.03090 & 12.4 & 8.2 & 14.9\end{array}$

$\begin{array}{lllllll}0.0300 & 200.0 & 0.6700 & 0.02970 & 13.2 & 10.1 & 16.6\end{array}$

$\begin{array}{lllllll}0.0300 & 400.0 & 0.2700 & 0.03220 & 13.5 & 9.9 & 16.7\end{array}$

$\begin{array}{lllllll}0.0300 & 400.0 & 0.4300 & 0.02930 & 13.1 & 8.2 & 15.4\end{array}$

$\begin{array}{lllllll}0.0300 & 400.0 & 0.6700 & 0.02890 & 13.7 & 10.2 & 17.0\end{array}$

$\begin{array}{lllllll}0.0300 & 800.0 & 0.4300 & 0.03910 & 17.2 & 10.3 & 20.1\end{array}$

$\begin{array}{lllllll}0.0300 & 800.0 & 0.6700 & 0.02280 & 18.3 & 11.6 & 21.6\end{array}$

$\begin{array}{lllllll}0.0300 & 1600.0 & 0.6700 & 0.02140 & 30.0 & 12.8 & 32.6\end{array}$ 


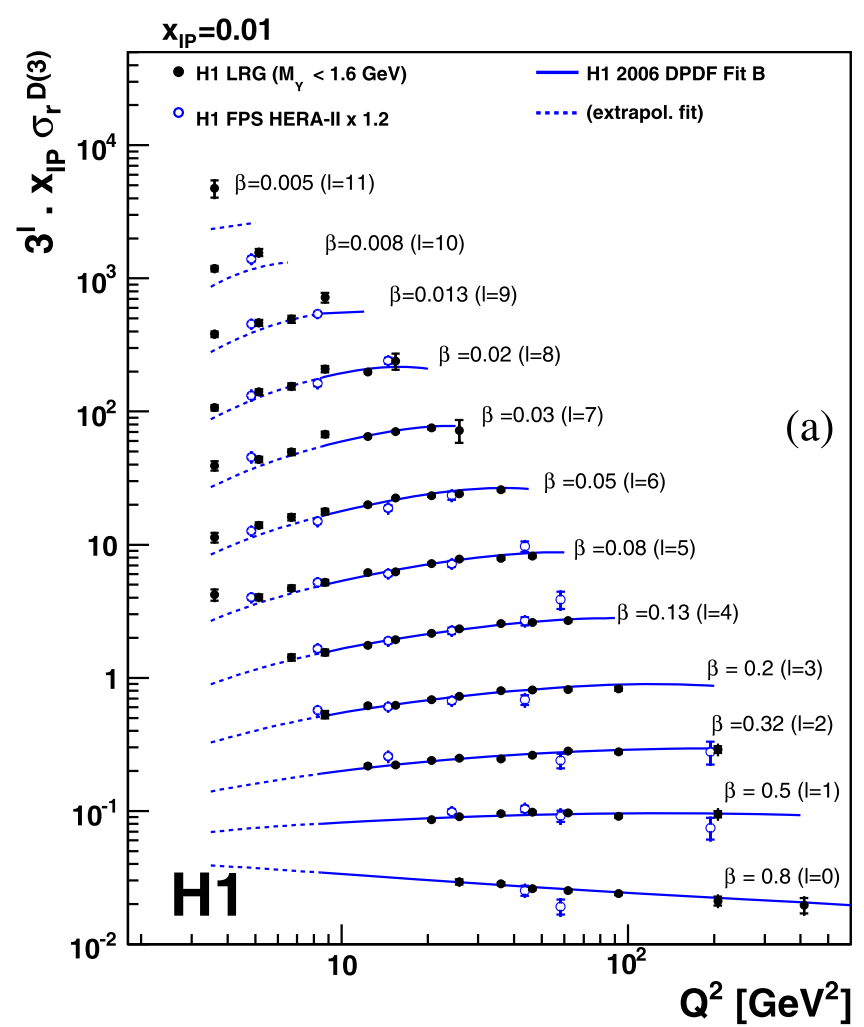

Fig. 9 The reduced diffractive cross section from combined H1 LRG data, multiplied by $x_{\mathbb{P}}$, at two fixed values of $x_{\mathbb{P}}=0.01$ (a) and 0.03 (b). The reduced cross section values are multiplied by a scaling factor $3^{l}$, with $l$ values as indicated in parentheses. The LRG data are compared with the H1 FPS results [12] interpolated to the LRG $\beta, Q^{2}$ and $x_{\mathbb{P}}$ values using a parametrisation of the H1 2006 DPDF Fit B [10].

\subsection{Ratio to inclusive DIS}

In analogy to hadronic scattering, the diffractive and the total cross sections can be related via the generalisation of the optical theorem to virtual photon scattering [65]. Many models of low $x$ DIS [66-71] assume links between these quantities. Comparing the $Q^{2}$ and $x$ dynamics of the diffractive with the inclusive cross section is therefore a powerful means of comparing the properties of the DPDFs with their inclusive counterparts and of testing models. The evolution of the diffractive reduced cross section with $Q^{2}$ can be compared with that of the inclusive DIS reduced cross section $\sigma_{r}$ by forming the ratio

$\frac{\sigma_{r}^{D(3)}\left(x_{\mathbb{P}}, x, Q^{2}\right)}{\sigma_{r}\left(x, Q^{2}\right)} \cdot(1-\beta) x_{\mathbb{P}}$,

at fixed $Q^{2}, \beta=x / x_{\mathbb{P}}$ and $x_{\mathbb{P}}$. A parametrisation of $\sigma_{r}$ from [72] is used. This quantity is equivalent to the ratio of diffractive to $\gamma^{*} p$ cross sections,

$\frac{M_{X}^{2} \frac{\mathrm{d} \sigma_{r}^{D(3)}\left(M_{X}, W, Q^{2}\right)}{\mathrm{d} M_{X}}}{\sigma_{\text {incl. }}^{\gamma^{*} p}\left(W, Q^{2}\right)}$,

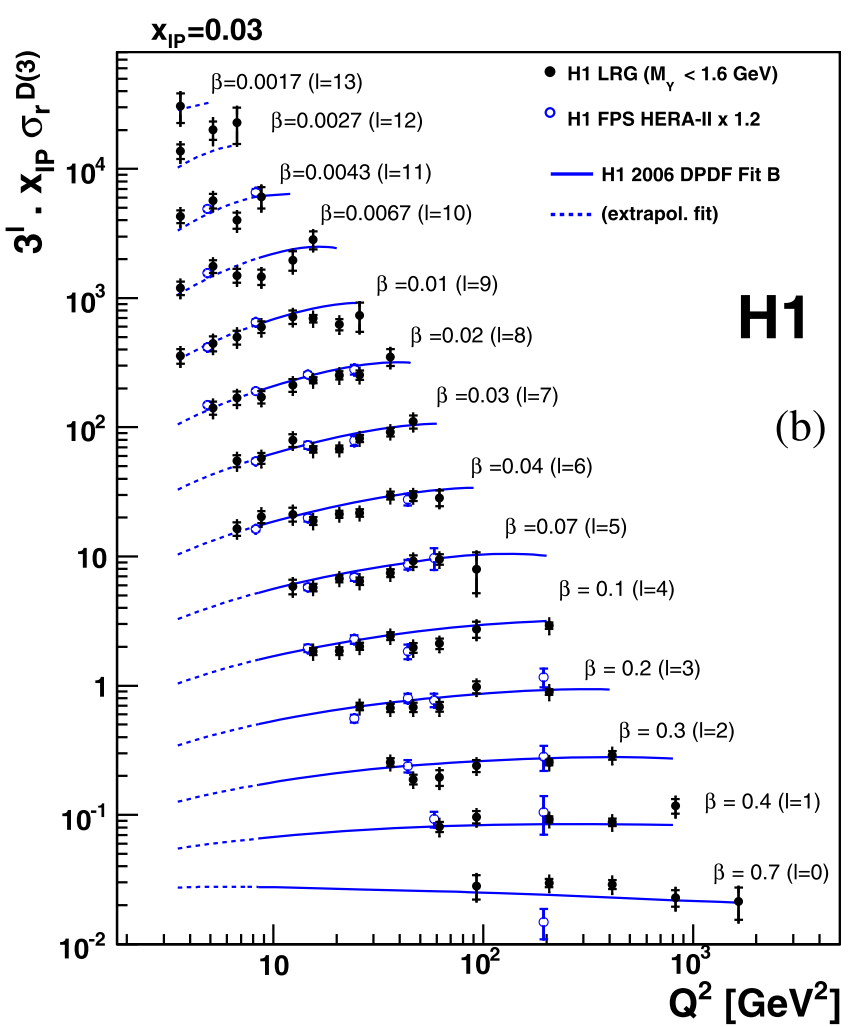

The FPS data are multiplied by a factor 1.2 (see Sect. 4.2). The overall normalisation uncertainties of $4 \%$ and $6 \%$ on the LRG and FPS data, respectively, are not shown. The measurements are displaced horizontally for better visibility. More details are explained in the caption of Fig. 3

studied in $[8,9,13]$ as a function of $W$ and $Q^{2}$ in ranges of $M_{X}$. Assuming proton vertex factorization in the DPDF approach, this ratio is expected to be independent of $Q^{2}$ and depends only weakly on $\beta$ and $x \simeq Q^{2} / W^{2}$ for sufficiently large $M_{X}$. A remaining weak $x$ dependence of the ratio may arise due to deviations from unity of the intercept of the Pomeron trajectory, which are studied in the next section. The ratio (10) is shown in Fig. 11 as a function of $x$ at fixed $x_{\mathbb{P}}$ and $Q^{2}$ values. The ratio of the diffractive to the inclusive cross section is found to be approximately constant with $x$ at fixed $Q^{2}$ and $x_{\mathbb{P}}$ except towards larger $x$ values which correspond to large $\beta$ values. This indicates that the ratio of quark to gluon distributions is similar in the diffractive and inclusive process when considered at the same low $x$ value. The ratio is also larger at high values of $x_{\mathbb{P}}, x_{\mathbb{P}}=0.03$, where the sub-leading exchange contribution of the diffractive cross section is not negligible, but it remains approximately constant with $x$. These observations are in agreement with previous similar studies [12]. The general behaviour of the ratio, and especially its decrease towards larger $x$, is reproduced by both the DPDF [10] and dipole model [16] predictions. 

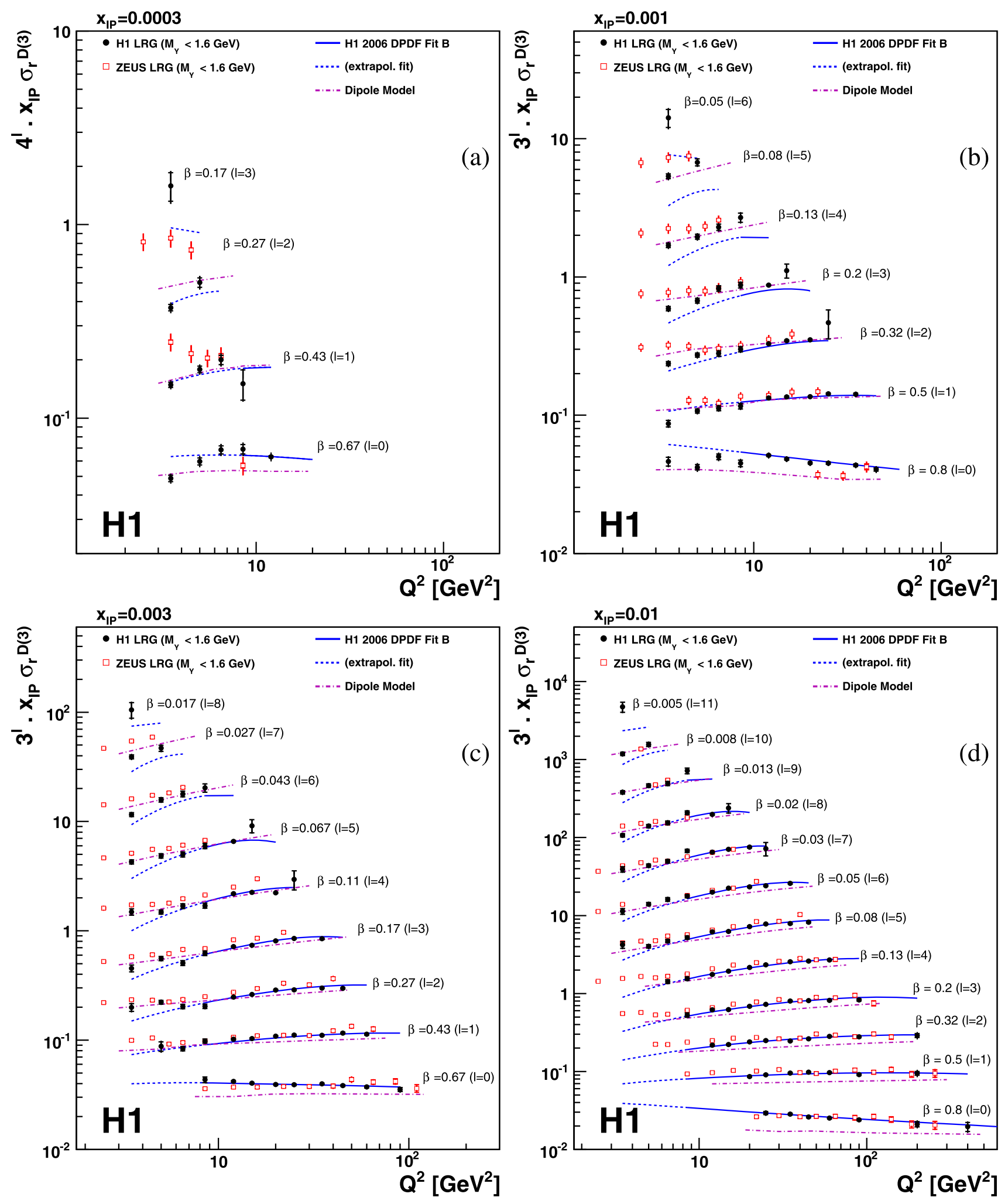

Fig. 10 The $Q^{2}$ dependence of the reduced diffractive cross section from combined $\mathrm{H} 1$ data, multiplied by $x_{\mathbb{P}}$, at different fixed values of $x_{\mathbb{P}}=0.0003(\mathbf{a}), 0.001(\mathbf{b}), 0.003(\mathbf{c})$ and $0.01(\mathbf{d})$. The present data are compared with the results of the ZEUS Collaboration [14], corrected to $M_{Y}<1.6 \mathrm{GeV}$ (see text). The $8 \%$ overall uncertainty on this

correction for ZEUS data is not shown. The overall normalisation uncertainties of $4 \%$ and $2.25 \%$ for the $\mathrm{H} 1$ and ZEUS data, respectively, are also not shown. Predictions from the H1 2006 DPDF Fit B [10] and dipole model [16] are displayed. More details are explained in the captions of Figs. 3 and 7 


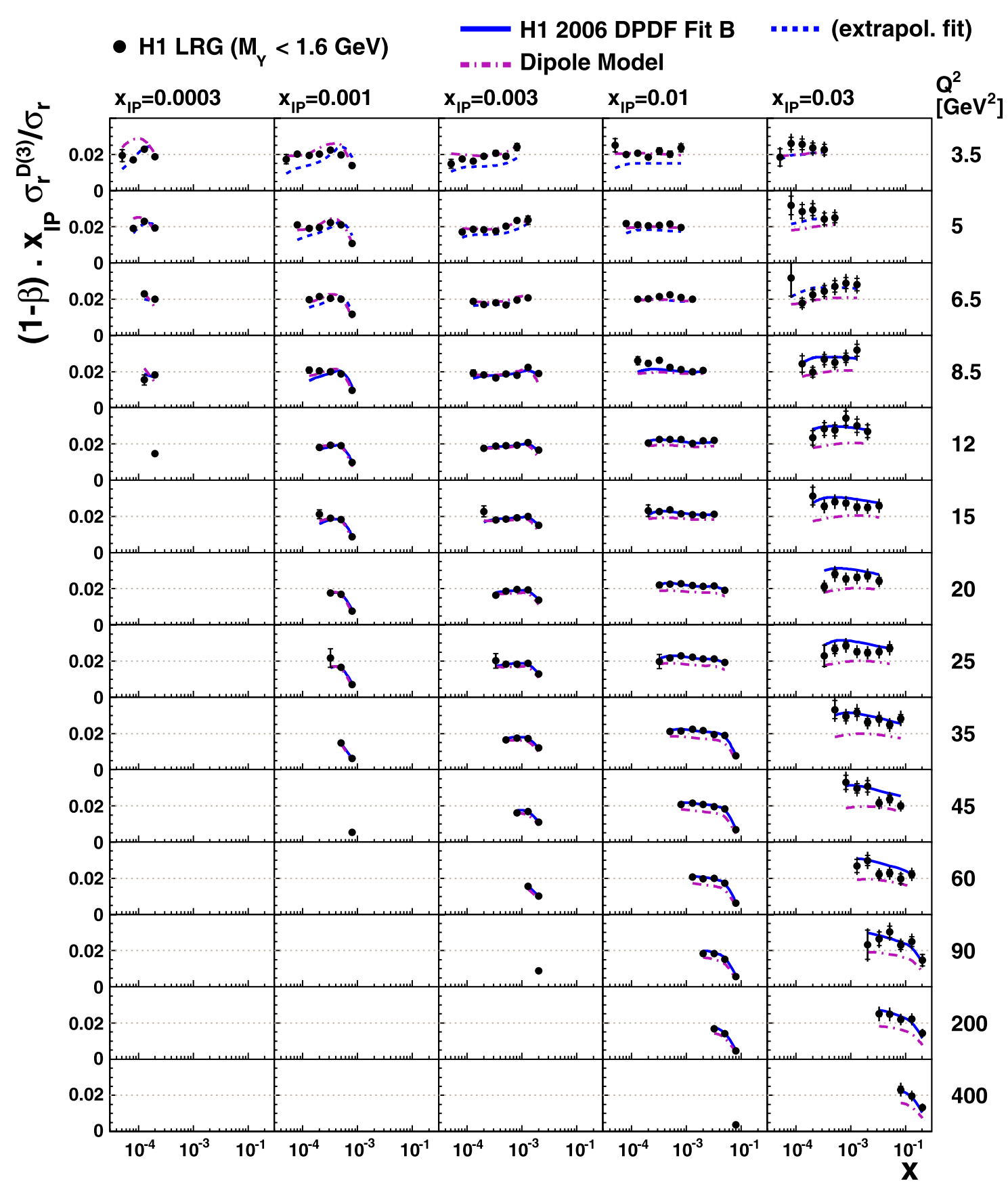

Fig. 11 The ratio of the diffractive to the inclusive reduced cross section, multiplied by $(1-\beta) x_{\mathbb{P}}$. The inner and outer error bars on the data points represent the statistical and total uncertainties, respectively.
The overall normalisation uncertainty of $4 \%$ is not shown. The curves are explained in the captions of Figs. 3 and 10

phenomenology and is usually expressed as a sum of two factorised contributions corresponding to Pomeron and secondary Reggeon trajectories

$$
\begin{aligned}
F_{2}^{D(3)}\left(Q^{2}, \beta, x_{\mathbb{P}}\right)= & f_{\mathbb{P} / p}\left(x_{\mathbb{P}}\right) F_{2}^{\mathbb{P}}\left(Q^{2}, \beta\right) \\
& +n_{\mathbb{R}} f_{\mathbb{R} / p}\left(x_{\mathbb{P}}\right) F_{2}^{\mathbb{R}}\left(Q^{2}, \beta\right) .
\end{aligned}
$$


In this parametrisation, $F_{2}^{\mathbb{P}}$ can be interpreted as the Pomeron structure function and $F_{2}^{\mathbb{R}}$ as an effective Reggeon structure function. The global normalisation of this last contribution is denoted $n_{\mathbb{R}}$. The Pomeron and Reggeon fluxes are assumed to follow a Regge behaviour with linear trajectories $\alpha_{\mathbb{P}, \mathbb{R}}(t)=\alpha_{\mathbb{P}, \mathbb{R}}(0)+\alpha_{\mathbb{P}, \mathbb{R}}^{\prime} t$, such that

$f_{\mathbb{P} / p, \mathbb{R} / p}\left(x_{\mathbb{P}}\right)=\int_{t_{\mathrm{cut}}}^{t_{\min }} \frac{e^{B_{\mathbb{P}, \mathbb{R}} t}}{x_{\mathbb{P}}^{2 \alpha \mathbb{P}, \mathbb{R}(t)-1}} \mathrm{~d} t$.

In this formula, $\left|t_{\min }\right|$ is the minimum kinematically allowed value of $|t|$ and $t_{\mathrm{cut}}=-1 \mathrm{GeV}^{2}$ is the limit of the measurement.

In Eq. (12), the values of $F_{2}^{\mathbb{P}}$ are treated as free parameters at each $\beta$ and $Q^{2}$ point, together with the Pomeron intercept $\alpha_{\mathbb{P}}(0)$ and the normalisation $n_{\mathbb{R}}$ of the sub-leading exchange. The values of the other parameters are fixed in the fit. The parameters $\alpha_{\mathbb{P}}^{\prime}=0.04_{-0.06}^{+0.08} \mathrm{GeV}^{-2}$ and $B_{\mathbb{P}}=5.7_{-0.9}^{+0.8}$ $\mathrm{GeV}^{-2}$ are taken from the last H1 FPS publication [12]. The intercept of the sub-leading exchange $\alpha_{\mathbb{R}}(0)=0.5 \pm 0.1$ is taken from [4]. The parameters $\alpha_{\mathbb{R}}^{\prime}=0.30_{-0.3}^{+0.6} \mathrm{GeV}^{-2}$ and $B_{\mathbb{R}}=1.6_{+0.4}^{-1.6} \mathrm{GeV}^{-2}$ are obtained from a parametrisation of previously published H1 FPS data [11]. Since the subleading exchange is poorly constrained by the data, values of $F_{2}^{\mathbb{R}}\left(Q^{2}, \beta\right)$ are taken from a parametrisation of the pion structure function [73], with a single free normalisation $n_{\mathbb{R}}$. Choosing a different parametrisation for the pion structure function [74] does not affect the results significantly.

In previous publications $[4,11,12,14]$, it has already been shown that fits of this form provide a good description of the data. This supports the proton vertex factorisation hypothesis whereby the $x_{\mathbb{P}}$ and $t$ dependences are decoupled from the $Q^{2}$ and $\beta$ dependences for each of the Pomeron and sub-leading contributions. This global conclusion can be refined using the advantage of the improved statistical precision of the present analysis. In the following, the full range in $Q^{2}$ is divided into six intervals: $Q^{2} \leq 6.5 \mathrm{GeV}^{2}, 6.5<$ $Q^{2} \leq 12 \mathrm{GeV}^{2}, 12<Q^{2} \leq 25 \mathrm{GeV}^{2}, 25<Q^{2} \leq 45 \mathrm{GeV}^{2}$, $45<Q^{2} \leq 90 \mathrm{GeV}^{2}$ and $Q^{2}>90 \mathrm{GeV}^{2}$. For each interval $i$, a free Pomeron intercept $\alpha_{\mathbb{P}}(0)\left[Q_{i}^{2}\right]$ is introduced. Thus the factorisation assumption can be tested differentially in $Q^{2}$ by allowing for a $Q^{2}$ dependence of the Pomeron intercept in the fit procedure. In the minimisation procedure the error of each data points is obtained by adding in quadrature the statistical and uncorrelated systematic uncertainties. The effect of correlated uncertainties is taken into account by repeating the fit multiple times with each correlated systematic error shifted by one standard deviation. The kinematic domain of the fit procedure is defined as $M_{X}>2$ $\mathrm{GeV}$ and $\beta<0.8$, in order to avoid resonances and potential higher-twist effects. This leads to 175 diffractive structure function values. The fit provides a good description of the data $\left(\chi^{2}=201\right)$. The results on the Pomeron intercept are presented in Fig. 12. No significant $Q^{2}$ dependence of the

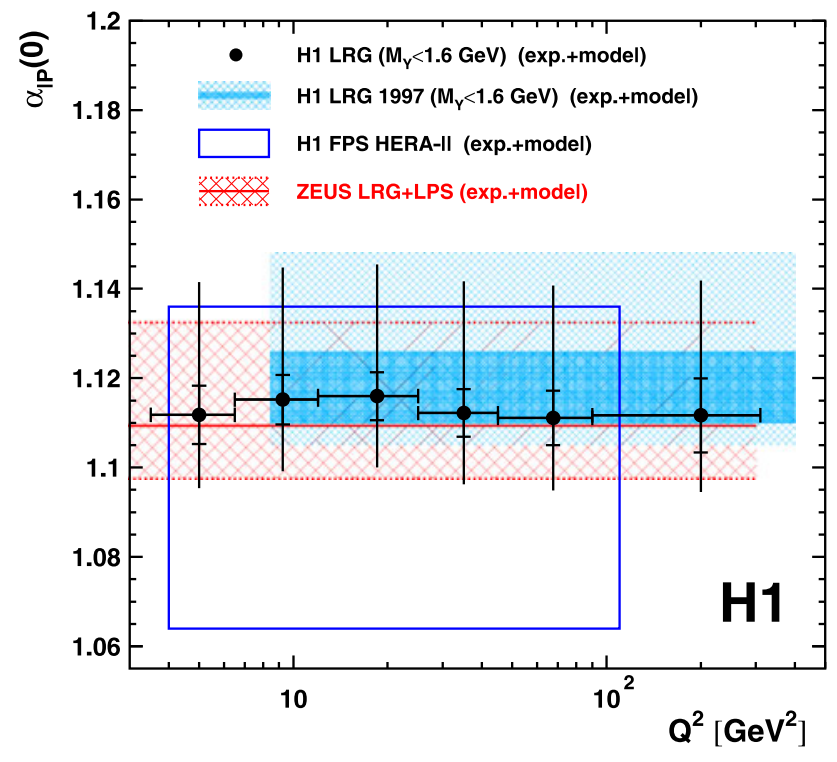

Fig. 12 Pomeron intercept values obtained from Regge fits in different $Q^{2}$ bins, as defined in the text (dots). The inner error bars represent the statistical and systematic errors added in quadrature and the outer error bars include model uncertainties in addition (see text for details). Previous determinations of the Pomeron intercept [10-12, 14] are also displayed for comparison. For these previous results the bands or boxes represent the combination of experimental and model uncertainties, always dominated by the model error

Pomeron intercept is observed, which supports the proton vertex factorisation hypothesis. The average value is found to be

$\alpha_{\mathbb{P}}(0)=1.113 \pm 0.002$ (exp. $)_{-0.015}^{+0.029}$ (model),

where the first error is the full experimental uncertainty and the second error expresses the model dependent uncertainty arising dominantly from the variation of $\alpha_{\mathbb{P}}^{\prime}$, which is strongly positively correlated with $\alpha_{\mathbb{P}}(0)$. As diffractive structure function values are determined with an assumption on $F_{L}^{D(3)}$, the influence of neglecting the $F_{L}^{D(3)}$ contribution is also included in the model dependent uncertainty. It gives rise to only a small effect. This is verified by repeating the fit procedure under the condition that data points with $y>0.45$ are excluded from the minimisation procedure, in order to reduce the impact of the $F_{L}^{D(3)}$ contribution. The number of data points is then reduced to 138 and the results are found to be the same as those of Fig. 12 within the statistical precision.

As illustrated in Fig. 12, the average $\alpha_{\mathbb{P}}(0)$ value obtained in this analysis together with the absence of a $Q^{2}$ dependence within the statistical precision of the measurement is in very good agreement with previous determinations in diffractive DIS [10-12, 14]. It also agrees within errors with a result obtained in diffractive photoproduction [76]. 


\section{Conclusions}

A measurement of the reduced inclusive diffractive cross section $\sigma_{r}{ }^{D(3)}\left(Q^{2}, \beta, x_{\mathbb{P}}\right)$ for the process $e p \rightarrow e X Y$ with $M_{Y}<1.6 \mathrm{GeV}$ and $|t|<1 \mathrm{GeV}^{2}$ is presented. New results are obtained using high statistics data taken from 1999 to 2007 by the H1 detector at HERA. These measurements are combined with previous $\mathrm{H} 1$ results obtained using the same technique for the selection of large rapidity gap events. The combined data span more than two orders of magnitude in $Q^{2}$ from $3.5 \mathrm{GeV}^{2}$ to $1600 \mathrm{GeV}^{2}$ and cover the range $0.0017 \leq \beta \leq 0.8$ for five fixed values of $x_{\mathbb{P}}$ in the range $0.0003 \leq x_{\mathbb{P}} \leq 0.03$. In the best measured region for $Q^{2} \geq 12 \mathrm{GeV}^{2}$, the statistical and systematic uncertainties are at the level of $1 \%$ and $5 \%$, respectively, with an additional overall normalisation uncertainty of $4 \%$. By comparing to the proton-tagged cross section measurements, a contribution of $20 \%$ of proton dissociation is found to be present in large rapidity gap data.

The combined $\mathrm{H} 1$ diffractive cross section measurements are compared with predictions from dipole and DPDF approaches. A reasonable description of the data is achieved by both models. The predictions of the dipole model, including saturation, can describe the low $Q^{2}$ kinematic domain of the measurements better than the previous H1 DPDF fits.

The ratio of the diffractive to the inclusive ep cross section is measured as a function of $x, Q^{2}$ and $x_{\mathbb{P}}$. At fixed $x_{\mathbb{P}}$ the ratio depends only weakly on $x$, except at the highest $x$ values. Proton PDF and dipole model predictions reproduce the behaviour of the ratio. This result implies that the ratio of quark to gluon distributions is similar in the diffractive and inclusive process when considered at the same low $x$ value.

The $x_{\mathbb{P}}$ dependence of $\sigma_{r}^{D(3)}\left(Q^{2}, \beta, x_{\mathbb{P}}\right)$ is described using a model motivated by Regge phenomenology, in which a leading Pomeron and a sub-leading exchange contribute. With the high statistics of the present analysis, it is possible to test for a possible $Q^{2}$ dependence of the Pomeron intercept with increased sensitivity. The results do not exhibit any dependence on $Q^{2}$. An average value of the effective Pomeron intercept over the full range in $Q^{2}$ can thus be obtained, which leads to $\alpha_{\mathbb{P}}(0)=1.113 \pm$ 0.002 (exp.) ${ }_{-0.015}^{+0.029}$ (model). This result is compatible with previous determinations and supports the proton vertex factorisation hypothesis.

\footnotetext{
Acknowledgements We are grateful to the HERA machine group whose outstanding efforts have made this experiment possible. We thank the engineers and technicians for their work in constructing and maintaining the $\mathrm{H} 1$ detector, our funding agencies for financial support, the DESY technical staff for continual assistance and the DESY directorate for the hospitality which they extend to the non-DESY members of the collaboration. We would like to thank C. Marquet for helpful discussions and for providing us with the dipole model predictions.
}

Open Access This article is distributed under the terms of the Creative Commons Attribution License which permits any use, distribution, and reproduction in any medium, provided the original author(s) and the source are credited.

\section{References}

1. M. Derrick et al. (ZEUS Collaboration), Phys. Lett. B 315, 481 (1993)

2. T. Ahmed et al. (H1 Collaboration), Nucl. Phys. B 429, 477 (1994)

3. T. Ahmed et al. (H1 Collaboration), Phys. Lett. B 348, 681 (1995). hep-ex/9503005

4. C. Adloff et al. (H1 Collaboration), Z. Phys. C 76, 613 (1997). hep-ex/9708016

5. M. Derrick et al. (ZEUS Collaboration), Z. Phys. C 68, 569 (1995). hep-ex/9505010

6. J. Breitweg et al. (ZEUS Collaboration), Eur. Phys. J. C 6, 43 (1999). hep-ex/9807010

7. S. Chekanov et al. (ZEUS Collaboration), Eur. Phys. J. C 25, 169 (2002). hep-ex/0203039

8. S. Chekanov et al. (ZEUS Collaboration), Eur. Phys. J. C 38, 43 (2004). hep-ex/0408009

9. S. Chekanov et al. (ZEUS Collaboration), Nucl. Phys. B 713, 3 (2005). hep-ex/0501060

10. A. Aktas et al. (H1 Collaboration), Eur. Phys. J. C 48, 715 (2006). hep-ex/0606004

11. A. Aktas et al. (H1 Collaboration), Eur. Phys. J. C 48, 749 (2006). hep-ex/0606003

12. F.D. Aaron et al. (H1 Collaboration), Eur. Phys. J. C 71, 1578 (2011). arXiv:1010.1476

13. S. Chekanov et al. (ZEUS Collaboration), Nucl. Phys. B 800, 1 (2008). arXiv:0802.3017

14. S. Chekanov et al. (ZEUS Collaboration), Nucl. Phys. B 816, 1 (2009). arXiv:0812.2003

15. F.D. Aaron et al. (H1 Collaboration), Eur. Phys. J. C 72, 1836 (2012). arXiv: 1107.3420

16. C. Marquet, Phys. Rev. D 76, 094017 (2007). arXiv:0706.2682

17. J. Collins, Phys. Rev. D 57, 3051 (1998). Erratum-ibid. D 61, 019902 (2000). hep-ph/9709499

18. L. Trentadue, G. Veneziano, Phys. Lett. B 323, 201 (1994)

19. G. Ingelman, P. Schlein, Phys. Lett. B 152, 256 (1985)

20. A. Donnachie, P. Landshoff, Phys. Lett. B 191, 309 (1987). [Erratum-ibid. B 198, 590 (1987)]

21. E. Feinberg, I. Pomeranchuk, Suppl. Nuovo Cimento 3, 652 (1956)

22. V. Gribov, JETP Lett. 41, 667 (1961)

23. J. Breitweg et al. (ZEUS Collaboration), Eur. Phys. J. C 5, 41 (1998). hep-ex/9804013

24. L. Alvero, J.C. Collins, J. Terron, J.J. Whitmore, Phys. Rev. D 59, 074022 (1999). hep-ph/9805268

25. T. Gehrmann, W.J. Stirling, Z. Phys. C 70, 89 (1996). hep-ph/ 9503351

26. K. Golec-Biernat, J. Kwiecinski, Phys. Lett. B 353, 329 (1995). hep-ph/9504230

27. C. Royon et al., Phys. Rev. D 63, 074004 (2001). hep-ph/0010015

28. J. Lamouroux et al., Nucl. Phys. B 649, 312 (2003). hep-ph/ 0210101

29. C. Royon et al., Nucl. Phys. B 746, 15 (2006). hep-ph/0602228

30. C. Royon et al., Nucl. Phys. B 781, 1 (2007). hep-ph/0609291

31. A. Martin, M. Ryskin, G. Watt, Eur. Phys. J. C 37, 285 (2004). hep-ph/0406224

32. A. Martin, M. Ryskin, G. Watt, Eur. Phys. J. C 44, 69 (2005). hep-ph/0504132 
33. G. Watt, in Proc. of the Workshop on New Trends in HERA Physics, Ringberg Castle, Germany, ed. by G. Grindhammer et al. (2005), p. 303. hep-ph/0511333

34. S. Chekanov et al. (ZEUS Collaboration), Nucl. Phys. B 831, 1 (2010). arXiv:0911.4119

35. A.H. Mueller, Nucl. Phys. B 335, 115 (1990)

36. N.N. Nikolaev, B.G. Zakharov, Z. Phys. C 49, 607 (1991)

37. E. Iancu, K. Itakura, S. Munier, Phys. Lett. B 590, 199 (2004). hep-ph/0310338

38. K. Golec-Biernat, M. Wüsthoff, Phys. Rev. D 59, 014017 (1999). hep-ph/9807513

39. A.M. Staśto, K. Golec-Biernat, J. Kwieciński, Phys. Rev. Lett. 86, 596 (2001). hep-ph/0007192

40. C. Marquet, L. Schoeffel, Phys. Lett. B 639, 471 (2006). hep-ph/ 0606079

41. K.J. Golec-Biernat, M. Wusthoff, Phys. Rev. D 60, 114023 (1999). hep-ph/9903358

42. G. Soyez, Phys. Lett. B 655, 32 (2007). arXiv:0705.3672

43. I. Abt et al. (H1 Collaboration), Nucl. Instrum. Methods A 386, 310 (1997)

44. I. Abt et al. (H1 Collaboration), Nucl. Instrum. Methods A 386, 348 (1997)

45. R.D. Appuhn et al. (H1 SPACAL Group), Nucl. Instrum. Methods A 386, 397 (1997)

46. B. Andrieu et al. (H1 Calorimeter Group), Nucl. Instrum. Methods A 350, 57 (1994)

47. B. Andrieu et al. (H1 Calorimeter Group), Nucl. Instrum. Methods A 336, 499 (1993)

48. B. List, A. Mastroberardino DIFFVM, in Proc. of the Workshop on Monte Carlo Generators for HERA Physics, ed. by A. Doyle, G. Grindhammer, G. Ingelman, H. Jung (1999), p. 396. DESYPROC-1999-02

49. M. Peez, Ph.D. thesis, Univ. Lyon, 2003. DESY-THESIS-2003023, available at http://www-h1.desy.de/publications/theses_list. html

50. S. Hellwig, Dipl. thesis, Univ. Hamburg, 2004, available at. http:// www-h1.desy.de/publications/theses_list.html

51. R. Brun et al., CERN-DD/EE/14-1

52. H. Jung, Comput. Phys. Commun. 86, 147 (1995). See also http:// www.desy.de/ jung/rapgap.html
53. M. Bengtsson, T. Sjöstrand, Z. Phys. C 37, 465 (1988)

54. B. Andersson et al., Phys. Rep. 97, 31 (1983)

55. T. Sjöstrand, Comput. Phys. Commun. 82, 74 (1994)

56. A. Kwiatkowski, H. Spiesberger, H. Möhring, Comput. Phys. Commun. 69, 155 (1992)

57. A. Courau et al., in Proc. of the Workshop "Physics at HERA", DESY, vol. 2, ed. by W. Buchmüller, G. Ingelman (1991), p. 902

58. R. Engel, J. Ranft, Phys. Rev. D 54, 4244 (1996). hep-ph/9509373

59. G. Gustafson, Phys. Lett. B 175, 453 (1986)

60. L. Lonnblad, Comput. Phys. Commun. 71, 15 (1992)

61. The Combined Data Together with the Full Correlation Information is provided at URL http://www-h1.desy.de/publications/ H1publication.short_list.html

62. A. Glazov, AIP Conf. Proc. 792, 237 (2005)

63. F.D. Aaron et al. (H1 Collaboration), Eur. Phys. J. C 63, 625 (2009). arXiv:0904.0929

64. F.D. Aaron et al. (H1 and ZEUS Collaboration), J. High Energy Phys. 1001, 109 (2010). arXiv:0911.0884

65. A. Mueller, Phys. Rev. D 2, 2963 (1970)

66. W. Buchmüller, A. Hebecker, Phys. Lett. B 355, 573 (1995). hep-ph/9504374

67. W. Buchmüller, T. Gehrmann, A. Hebecker, Nucl. Phys. B 537, 477 (1999). hep-ph/9808454

68. A. Edin, G. Ingelman, J. Rathsman, Phys. Lett. B 366, 371 (1996). hep-ph/9508386

69. A. Edin, G. Ingelman, J. Rathsman, Z. Phys. C 75, 57 (1997). hep-ph/9605281

70. J. Rathsman, Phys. Lett. B 452, 364 (1999). hep-ph/9812423

71. S. Brodsky et al., Phys. Rev. D 71, 074020 (2005). hep-ph/ 0409119

72. F.D. Aaron et al. (H1 Collaboration), Eur. Phys. J. C 64, 561 (2009). arXiv:0904.3513

73. M. Glück, E. Reya, A. Vogt, Z. Phys. C 53, 651 (1992)

74. J. Owens, Phys. Rev. D 30, 943 (1984)

75. F.D. Aaron et al. (H1 Collaboration), Eur. Phys. J. C 68, 381 (2010). arXiv:1001.0532

76. C. Adloff et al. (H1 Collaboration), Z. Phys. C 74, 221 (1997). hep-ex/9702003 